\title{
Cubic interactions of massless higher spins in (A)dS: metric-like approach
}

\author{
Euihun JOUNG ${ }^{a}$ Massimo TARONNA $^{a}$ \\ ${ }^{a}$ Scuola Normale Superiore and INFN \\ Piazza dei Cavalieri 7, 56126 Pisa, Italy \\ E-mail: euihun.joung@sns.it, massimo.taronna@sns.it
}

\begin{abstract}
Cubic interactions of higher-spin gauge fields in $(A) d S_{d}$ are studied in the metric-like approach. Making use of the traceless and transverse constraints together with the ambient-space formalism, all consistent parity-invariant cubic vertices are obtained for $d \geq 4$ in a closed form pointing out the key role of their flat-space counterparts.
\end{abstract}




\section{Contents}

1 Introduction 1

2 Cubic interactions of HS gauge fields in flat space 4

2.1 Traceless and Transverse HS gauge fields 5

$\begin{array}{lll}2.2 & \text { General solutions for consistent cubic interactions } & 6\end{array}$

3 Free (A)dS HS gauge fields and Ambient-space formalism $\quad 7$

3.1 Traceless and Transverse HS gauge fields 8

3.2 Ambient space formalism $\quad 9$

4 Cubic interactions of HS gauge fields in (A)dS backgrounds 11

$\begin{array}{lll}4.1 \text { Construction of consistent cubic interactions } & 11\end{array}$

$\begin{array}{lll}4.2 & \text { General solutions for consistent cubic interactions } & 14\end{array}$

$\begin{array}{lll}4.3 & \text { Reduction to (A)dS-intrinsic expressions } & 17\end{array}$

$\begin{array}{llr}5 & \text { Discussion and Outlook } & 19\end{array}$

5.1 Relation to the Fradkin-Vasiliev vertices 20

$\begin{array}{lll}5.2 \text { Outlook } & 21\end{array}$

$\begin{array}{ll}\text { A Foregoing the traceless and transverse constraints } & 22\end{array}$

B Proof at the $\delta^{(2)}$ level $\quad 25$

C Radial reduction of the 3-3-2 vertex $\quad 25$ 


\section{Introduction}

Understanding the systematics of higher-spin (HS) gauge theories ${ }^{1}$ has been attracting an increasing attention in recent years, and finding a consistent Lagrangian that describes their interactions is one of the main problems in the subject. Vasiliev's equations $[10,11]$ provide at present the only known fully non-linear consistent description, at least at the classical level, of an infinite number of HS gauge fields of all spins. ${ }^{2}$ However the nature of their couplings still leaves interesting questions to be answered. The generalization of the lower-spin gauge interactions of Yang-Mills and Gravity to HS is associated with a non-linear deformation of the Abelian HS gauge symmetries of the free theory $[19,20]^{3}$ and can be studied perturbatively by means of the Noether procedure. This actually rests on enforcing gauge invariance of the full Lagrangian order by order in the number of fields, and has been considered by mainly two different perspectives: frame-like or metric-like formalisms.

Important progress on HS cubic interactions in the frame-like approach was obtained by Fradkin and Vasiliev (FV) [31, 32] who extended the gravitational minimal coupling to $s_{1}-s_{2}-s_{3}$ HS couplings. Their construction of cubic couplings is consistent in (Anti) de Sitter ((A)dS) backgrounds, and one of its essential features is the presence of inverse powers of the cosmological constant. Very recently, these interaction vertices in $A d S_{4}$ were generalized to $A d S_{d}[33]$, that were conjectured to cover all vertices that can be constructed in terms of connection one-forms and curvature two-forms of symmetric HS gauge fields. In fact, the goal of the present paper is the same as that of [33], and it would be in principle interesting to explore the relation of our results with those of [33]. This comparison is although non-trivial since the two constructions use very different mathematical devices, and we will only discuss in the conclusion how the FV structure of the vertices is recovered in our approach. See [34-37] for other recent developments in the frame-like approach to the cubic-interaction problem.

On the other hand, the flat-space cubic vertices of HS gauge fields in the metric-like formalism were investigated first by Berends, Burgers and van Dam [38, 39], and then by many other authors. ${ }^{4}$ Notably, the consistent vertices were classified by Metsaev [40-42] in terms of the number of derivatives within the lightcone approach. Despite various efforts made along the years by a number of authors, only recently has it been possible to arrive at a covariant description of all bosonic flat-space cubic interactions by Manvelyan, Mkrtchyan and Ruehl in [43-45] from a field theoretical perspective. At the same time, starting from a String Theory vantage point and with a careful analysis of the gauge invariant pieces contained in the string amplitudes, all consistent cubic interactions involving any bosonic and fermionic fields were obtained by Sagnotti and one of the authors in [46, 47]. These results pointed out the key role of on-shell expressions (the part of vertex that

\footnotetext{
${ }^{1}$ For some recent reviews of HS gauge theories, see e.g. the proceeding [1] (which includes contributions $[2-6])$ and $[7-9]$.

${ }^{2}$ See e.g. [5, 12] for some reviews of Vasiliev's equations, and [13-18] for a recent proposal on the action principle, observables and some exact solutions of Vasiliev's equations.

${ }^{3}$ See [21-27] for the unconstrained formulation of HS gauge theory, and [28-30] for recent developments.

${ }^{4}$ See e.g. the review [9] for an exhaustive list of works.
} 
does not involve divergences and traces ${ }^{5}$ of fields) of the cubic interactions from which one can recursively reconstruct any consistent off-shell cubic action. Further results and developments on higher-order vertices and scattering amplitudes can be found in [49] and [48].

The metric-like approach to (A)dS cubic vertices was also explored by some authors. For instance, the $3-3-2$ vertex was obtained in $[50,51]$, and the $s-0-0$ vertices were constructed in [52] by using the standard Noether procedure. The latter were also studied in [53] by using the BRST technique ${ }^{6}$, and also using current couplings in $[56,57] .{ }^{7}$

\section{Summary of results}

The aim of the present paper is to construct and classify the consistent parity-invariant cubic interactions of bosonic symmetric HS gauge fields in (A)dS backgrounds of any dimension greater than three within the metric-like formalism [62]. The expressions of flat-space cubic vertices in this formalism are highly involved ${ }^{8}$, and one can expect that the $(\mathrm{A}) \mathrm{dS}$ vertices may have even more complicated expressions. In order to circumvent the complexity, we first exploit the simplicity of working with the transverse and traceless (TT) constraints on the fields, which we expect to be systematically removable. Second, we employ the ambient-space description of (A)dS fields.

Transverse and Traceless constraints One of the main lessons in the recent construction of flat-space cubic interactions is that the full (off-shell) expressions of the vertices are fixed by their on-shell forms. The latter may be regarded as the consistent cubicinteraction vertices for the system of HS gauge fields with the TT constraints ${ }^{9}$. In this approach the kinetic term of HS fields $\varphi_{\mu_{1} \cdots \mu_{s}}$ becomes simply $\varphi^{\mu_{1} \cdots \mu_{s}} \partial^{2} \varphi_{\mu_{1} \cdots \mu_{s}}$, and is invariant under $\delta \varphi_{\mu_{1} \cdots \mu_{s}}=\partial_{\left(\mu_{1}\right.} \varepsilon_{\left.\mu_{2} \cdots \mu_{s}\right)}$ with the gauge parameters $\varepsilon_{\mu_{1} \cdots \mu_{s-1}}$ also subjected to TT constraints as well as an additional differential constraint $\partial^{2} \varepsilon_{\mu_{1} \cdots \mu_{s-1}}=0$. The key observation is that the consistent cubic-interaction problem can be addressed at this level, while foregoing the constraints requires a tedious but well defined procedure. Therefore, also for the (A)dS cubic interactions, we work within the TT setting where the transverse constraint is now with respect to the (A)dS-covariant derivative.

Ambient-space formalism Differently from the flat-space vertices, even after imposing the TT constraints the structure of $(\mathrm{A}) \mathrm{dS}$ cubic vertices is still highly non-trivial due to the non-commutativity of the covariant derivatives. A further simplification is achieved making use of the ambient-space formalism [62, 65-67] of (A)dS fields, where one rewrites intrinsic

\footnotetext{
${ }^{5}$ The traceless constraint is needed for the irreducibility of the representations. For a more detailed analysis of other possibilities, see [48].

${ }^{6}$ See also [54] for the general strategy of the construction, and see [55] for a review of the BRST approach.

${ }^{7}$ See $[58,59]$ for HS current exchanges, and see $[60,61]$ for flat-space current-coupling interactions and their application to the effective action in a HS background.

${ }^{8}$ See however $[47,48]$ and $[45,63]$ for more compact expressions.

${ }^{9}$ For completeness, let us mention that the TT decomposition has been introduced, although from a slightly different perspective, in the $s=2$ case [64].
} 
(A)dS quantities in terms of simpler flat-space ones. ${ }^{10}$ Recently, it was also exploited in order to construct spin-s gauge interactions with a scalar field [57].

The key feature of the ambient-space formalism is to regard the (A)dS space as the codimension-one hyper-surface $X^{2}=L^{2}$ in an ambient flat space-time parameterized by Cartesian coordinates $X^{M}$ with $M=0,1, \cdots, d$. In this formalism, the ambient-space HS fields $\Phi_{M_{1} \cdots M_{s}}$ that are homogeneous in $X^{M}$ and tangent to the hyper-surface, are in one-to-one correspondence to the (A)dS fields $\varphi_{\mu_{1} \cdots \mu_{s}}$. Moreover, the field equations and gauge transformations, as well as the TT constraints of (A)dS fields, can be derived from those of the ambient-space fields by a radial-dimensional reduction. The only subtlety of this formalism arises from the formally diverging radial integral at the level of the action. This can be cured with a $\delta$-function insertion of the form $\delta\left(\frac{\sqrt{X^{2}}}{L}-1\right)$. The presence of $\delta$ function is the main difference between the flat-space constructions and the ambient-space (A)dS ones. It requires particular care since it spoils the usual flat-space property that the integral of a total derivative vanishes.

With the aid of the TT constraints and the ambient-space formalism, the problem of finding consistent cubic interactions of HS fields in (A)dS becomes almost the same problem of flat-space vertices. The only difference, as we anticipated, is that the ambient-space action contains a $\delta$-function insertion, which makes the ambient-space total-derivative terms arising from gauge variations non-trivial. In order to compensate these gauge-variation terms, cubic-interaction vertices must also include proper total-derivative terms. To summarize, all consistent (A)dS cubic interactions read

$$
\begin{aligned}
& S^{(3)}=\frac{1}{3 !} \sum_{s_{1}, s_{2}, s_{3}=0}^{\infty} \sum_{n=0}^{\min \left\{\mathrm{s}_{1}, \mathrm{~s}_{2}, \mathrm{~s}_{3}\right\}} g_{a_{1} a_{2} a_{3}}^{s_{1} s_{2} s_{3}, n} \int \frac{d^{d+1} X}{L} \delta\left(\frac{\sqrt{X^{2}}}{L}-1\right) \times \\
& \times\left[\partial_{U_{1}} \cdot\left(\partial_{X_{23}}+\alpha \partial_{X}\right)\right]^{s_{1}-n}\left[-2 \partial_{U_{2}} \cdot\left(\partial_{X_{1}}-\frac{\alpha-1}{\alpha+1} \partial_{X}\right)\right]^{s_{2}-n}\left[2 \partial_{U_{3}} \cdot\left(\partial_{X_{1}}-\frac{\alpha+1}{\alpha-1} \partial_{X}\right)\right]^{s_{3}-n} \\
& \times\left[\partial_{U_{2}} \cdot \partial_{U_{3}} \partial_{U_{1}} \cdot\left(\partial_{X_{23}}+\beta \partial_{X}\right)-2 \partial_{U_{3}} \cdot \partial_{U_{1}} \partial_{U_{2}} \cdot\left(\partial_{X_{1}}+\frac{\alpha-\beta}{\alpha+1} \partial_{X}\right)\right. \\
& \left.\quad+2 \partial_{U_{1}} \cdot \partial_{U_{2}} \partial_{U_{3}} \cdot\left(\partial_{X_{1}}+\frac{\alpha-\beta}{\alpha-1} \partial_{X}\right)\right]\left.^{n} \Phi^{a_{1}}\left(X_{1}, U_{1}\right) \Phi^{a_{2}}\left(X_{2}, U_{2}\right) \Phi^{a_{3}}\left(X_{3}, U_{3}\right)\right|_{\substack{x_{1}=X_{2}=X_{3}=X \\
U_{1}=U_{2}=U_{3}=0}},
\end{aligned}
$$

where the form of the vertices is encoded in a differential operator acting on the generating function of ambient-space fields:

$$
\Phi^{a}(X, U)=\sum_{s=0}^{\infty} \frac{1}{s !} \Phi_{M_{1} \cdots M_{s}}^{a}(X) U^{M_{1}} \cdots U^{M_{s}},
$$

while $\partial_{X^{M}}=\partial_{X_{1}^{M}}+\partial_{X_{2}^{M}}+\partial_{X_{3}^{M}}$ is the total derivative, and $\partial_{X_{i j}^{M}}:=\partial_{X_{i}^{M}}-\partial_{X_{j}^{M}}$. Different choices of $\alpha$ and $\beta$ can be in fact absorbed into the coupling constants, and hence one can work with any particular choice. The number of ambient-space derivatives in (1.1) is

$$
\Delta=s_{1}+s_{2}+s_{3}-2 n,
$$

but, when radially reduced, different pieces of the (A)dS vertices involve different number of covariant derivatives: $\Delta, \Delta-2, \ldots, 1$ (or 0 ), while whenever the number of derivatives

\footnotetext{
10 The ambient-space formalism has been used for a large number of applications. See e.g. [68-75].
} 
decreases by two the corresponding mass-dimension is compensated by the cosmological constant $\Lambda:=1 / L^{2} .{ }^{11}$ This structure makes clear the relation of (1.1) to the FV vertices, where the inverse-power expansion in $\Lambda$ appears. For instance, concentrating on the gravitational couplings $\left(s_{1}=s_{2}=s\right.$ and $\left.s_{3}=n=2\right)$ in (1.1), the action can be recast in terms of an inverse-power series in $\Lambda$ as

$$
S^{(2)}+S^{(3)}=\frac{\lambda_{s}}{G} \sum_{r=2}^{s} \frac{1}{\Lambda^{r-2}} \int_{(A) d S_{d}} \mathcal{L}_{r} .
$$

In order to get this expression, we made use of the redefinitions $g^{s s 2,2}=\Lambda^{2-s} \sqrt{G} \lambda_{s}$ and $\varphi^{(s)}=\phi^{(s)} / \sqrt{G}$ with the gravitational coupling constant $G . \mathcal{L}_{r}$ 's are cubic vertices which are separately gauge invariant under the spin 2 gauge transformation and can be written schematically as

$$
\mathcal{L}_{r}=D^{2(r-1)} h \phi^{(s)} \phi^{(s)}+\Lambda D^{2(r-2)} h \phi^{(s)} \phi^{(s)},
$$

where $\mathcal{L}_{2}$ should involve the gravitational minimal coupling. Notice as well that the inversepower $\Lambda$-expansion has its origin from the redefinition of the coupling constant $g^{s s 2,2}$, which makes the two-derivative part of the vertex independent of $\Lambda$. A particular flat-space limit of the FV vertices considered by Boulanger, Leclercq and Sundell [51] is also discussed along the same lines.

The outline of this paper is as follows. In Section 2, we review the consistent flat-space cubic vertices, which will play an important role in the (A)dS case. In Section 3, we define the TT-constrained system of free HS gauge fields in (A)dS, and reformulate it in the ambient-space formalism. Section 4 is the main part of the paper, where we construct the consistent cubic interactions in (A)dS within the TT and ambient-space formalisms. We show also how to get the (A)dS intrinsic expressions by radially reducing the ambient-space ones. Section 5 contains discussions of our results. In Appendix A we review how to forego the constraints for the case of flat-space vertices and then discuss the (A)dS cases. Finally, Appendices B and C contain some technical details on our constructions.

\section{Cubic interactions of HS gauge fields in flat space}

Let us begin with reviewing the recent results on flat-space cubic vertices. The construction of on-shell cubic vertices is relatively simple, and the corresponding off-shell versions can be systematically recovered. Notice that the construction of flat-space cubic vertices with the TT constraints can be viewed as the covariant version of Metsaev's lightcone result [41].

In order to distinctly separate the initial construction and the later completion of vertices, we slightly change our viewpoint and begin with a system of HS fields subject to the traceless and transverse (TT) constraints. This is nonetheless on its own a consistent setting to search for consistent deformations of the free theory. In this section, we first

\footnotetext{
${ }^{11}$ The correct relation between the cosmological constant $\Lambda_{\text {C.C. }}$ and the radius of (A)dS is $\Lambda_{\text {C.C. }}=(d-1)(d-2) /\left(2 L^{2}\right)=\Lambda(d-1)(d-2) / 2$. However in this paper, for simplicity, we call also $\Lambda$ cosmological constant.
} 
formulate the free HS gauge theory as a TT-constrained system and then construct the consistent cubic interactions for that system.

\subsection{Traceless and Transverse HS gauge fields}

In order to work with compact expressions, we resort to generating functions of symmetric tensor fields, where the HS fields $\varphi_{\mu_{1} \cdots \mu_{s}}$ are contracted with auxiliary variables $u^{\mu}$ as

$$
\varphi(x, u):=\sum_{s=0}^{\infty} \frac{1}{s !} \varphi_{\mu_{1} \cdots \mu_{s}}(x) u^{\mu_{1}} \cdots u^{\mu_{s}} .
$$

In this notation the TT constraints can be written as

$$
\partial_{u}^{2} \varphi=0, \quad \partial_{u} \cdot \partial_{x} \varphi=0 .
$$

For this constrained system of fields, the quadratic action does not allow any non-trivial tensor contraction and is given by a scalar-field-like action of the form:

$$
S^{(2)}=\frac{1}{2} \int d^{d} x\left[\delta_{a_{1} a_{2}} e^{\partial_{u_{1}} \cdot \partial_{u_{2}}} \varphi^{a_{1}}\left(x_{1}, u_{1}\right) \partial_{x_{2}}^{2} \varphi^{a_{2}}\left(x_{2}, u_{2}\right)\right]_{\substack{x_{i}=x \\ u_{i}=0}}
$$

Here we have introduced colors for HS fields, labeled by $a_{i}$, for the description of YangMills-like interactions associated to Chan-Paton factors. It is useful to notice that the field equations for this system simply reads

$$
\partial_{x}^{2} \varphi \approx 0
$$

where $\approx$ means herefrom that the equality holds modulo the free field equation. This action is invariant under the usual linearized gauge transformations:

$$
\delta_{\varepsilon}^{(0)} \varphi=u \cdot \partial_{x} \varepsilon .
$$

Due to the TT constraints of the fields, the gauge parameters are constrained as well to satisfy the TT constraints:

$$
\partial_{u}^{2} \varepsilon=0, \quad \partial_{u} \cdot \partial_{x} \varepsilon=0,
$$

and also one additional field-equation-like differential constraint:

$$
\partial_{x}^{2} \varepsilon=0 .
$$

In fact, the latter constraints enable one to forego the TT constraints order by order in the number of divergences and traces.

As one can see, the TT constraints are closely related to the representations of the Lorentz group. In this respect, the transverse constraint can be considered as the condition to deal with a general representation of the Lorentz group while the traceless constraint selects irreducible ones. We keep the TT constraints for gauge fields (2.2) and gauge parameters (2.6) at the interacting level, but the gauge transformations (2.5) and the last constraint of gauge parameters (2.7) will be deformed by cubic-interaction vertices. 


\subsection{General solutions for consistent cubic interactions}

In this section we review the construction of the consistent parity-invariant cubic interactions for $d \geq 4$ that deform the free HS gauge theory. We begin with the most general expression for a cubic interaction which, in terms of generating functions, can be written in the form: ${ }^{12}$

$$
\begin{aligned}
S^{(3)}=\frac{1}{3 !} \int d^{d} x & C_{a_{1} a_{2} a_{3}}\left(\partial_{x_{1}}, \partial_{x_{2}}, \partial_{x_{3}} ; \partial_{u_{1}}, \partial_{u_{2}}, \partial_{u_{3}}\right) \times \\
& \times\left.\varphi^{a_{1}}\left(x_{1}, u_{1}\right) \varphi^{a_{2}}\left(x_{2}, u_{2}\right) \varphi^{a_{3}}\left(x_{3}, u_{3}\right)\right|_{\substack{x_{i}=x \\
u_{i}=0}},
\end{aligned}
$$

where $C_{a_{1} a_{2} a_{3}}$ satisfies the symmetry properties:

$$
C_{a_{2} a_{1} a_{3}}(2,1,3)=C_{a_{1} a_{3} a_{2}}(1,3,2)=C_{a_{1} a_{2} a_{3}}(1,2,3) .
$$

Taking into account integrations by parts and the TT constraints (2.2), we can conclude that $C_{a_{1} a_{2} a_{3}}$ depends in principle on all possible Lorentz invariants for the parity-invariant interactions $^{13}$ as

$$
C_{a_{1} a_{2} a_{3}}\left(\mathcal{Y}_{1}, \mathcal{Y}_{2}, \mathcal{Y}_{3}, \mathcal{Z}_{1}, \mathcal{Z}_{2}, \mathcal{Z}_{3}\right)
$$

where

$$
\begin{aligned}
& \mathcal{Y}_{1}:=\partial_{u_{1}} \cdot \partial_{x_{23}}, \quad \mathcal{Y}_{2}:=\partial_{u_{2}} \cdot \partial_{x_{31}}, \quad \mathcal{Y}_{3}:=\partial_{u_{3}} \cdot \partial_{x_{12}}, \\
& \mathcal{Z}_{1}:=\partial_{u_{2}} \cdot \partial_{u_{3}}, \quad \mathcal{Z}_{2}:=\partial_{u_{3}} \cdot \partial_{u_{1}}, \quad \mathcal{Z}_{3}:=\partial_{u_{1}} \cdot \partial_{u_{2}} \text {. }
\end{aligned}
$$

Here we made use of the compact notation $\partial_{x_{i j}^{\mu}}:=\partial_{x_{i}^{\mu}}-\partial_{x_{j}^{\mu}}$. This choice makes it possible to get rid of the ambiguity related to the total derivative terms. Notice that we have excluded terms proportional to $\partial_{x_{i}} \cdot \partial_{x_{j}}$ 's since they are proportional to the field equations up to a total derivative term, so that they can be removed by proper field redefinitions.

The Noether procedure, the order by order gauge invariance of the action, gives at the cubic level

$$
\delta^{(1)} S^{(2)}+\delta^{(0)} S^{(3)}=0 \quad \Rightarrow \quad \delta^{(0)} S^{(3)} \approx 0 .
$$

This, in our notation for $d \geq 4^{14}$, is equivalent to

$$
\left.\left[C_{a_{1} a_{2} a_{3}}, u_{i} \cdot \partial_{x_{i}}\right](\cdots)\right|_{u_{i}=0} \approx(\text { total derivative }) .
$$

Each $C_{a_{1} a_{2} a_{3}}$ will define a proper nonlinear deformation (to the first order in the fields) of the linear gauge symmetries. Making use of the identities

$$
\left[\mathcal{Y}_{i}, u_{j} \cdot \partial_{x_{j}}\right] \approx(\text { total derivative }), \quad\left[\mathcal{Z}_{i}, u_{j} \cdot \partial_{x_{j}}\right]=-\frac{1}{2} \epsilon_{i j k} \mathcal{Y}_{k}+(\text { total derivative }),
$$

with the antisymmetric Levi-Civita symbol $\epsilon_{i j k}$, the gauge consistency condition (2.13) gives the following differential equation for $C_{a_{1} a_{2} a_{3}}$ of (2.10) and its cyclic permutations on $(1)(2)(3)$ :

$$
\left(\mathcal{Y}_{1} \partial_{\mathcal{Z}_{2}}-\mathcal{Y}_{2} \partial_{\mathcal{Z}_{1}}\right) C_{a_{1} a_{2} a_{3}}\left(\mathcal{Y}_{i}, \mathcal{Z}_{i}\right)=0
$$

\footnotetext{
${ }^{12}$ For example, $\partial^{\nu} \varphi_{\mu_{1} \mu_{2}} \partial^{\rho_{1}} \varphi_{\nu}^{\mu_{1} \mu_{2} \rho_{2}} \varphi_{\rho_{1} \rho_{2}}$ is given by $C=\partial_{x_{1}} \cdot \partial_{u_{2}} \partial_{x_{2}} \cdot \partial_{u_{3}}\left(\partial_{u_{1}} \cdot \partial_{u_{2}}\right)^{2} \partial_{u_{2}} \cdot \partial_{u_{3}}$.

13 One can consider as well the totally antisymmetric tensor $\epsilon_{\mu_{1} \cdots \mu_{d}}$ for the parity-violating interactions, which however do not exist for $d>7$. See [76] for parity-violating cubic vertices of spin 3 fields.

${ }^{14}$ In 3 dimension, one should explicitly take into account that the Weyl tensor vanishes.
} 
The general solution for these differential equations is given by

$$
C_{a_{1} a_{2} a_{3}}\left(\mathcal{Y}_{1}, \mathcal{Y}_{2}, \mathcal{Y}_{3}, \mathcal{Z}_{1}, \mathcal{Z}_{2}, \mathcal{Z}_{3}\right)=\mathcal{K}_{a_{1} a_{2} a_{3}}\left(\mathcal{Y}_{1}, \mathcal{Y}_{2}, \mathcal{Y}_{3}, \mathcal{G}\right)
$$

where the operator $\mathcal{G}$ is defined as

$$
\begin{aligned}
\mathcal{G} & :=\mathcal{Y}_{1} \mathcal{Z}_{1}+\mathcal{Y}_{2} \mathcal{Z}_{2}+\mathcal{Y}_{3} \mathcal{Z}_{3} \\
& =\partial_{u_{2}} \cdot \partial_{u_{3}} \partial_{u_{1}} \cdot \partial_{x_{23}}+\partial_{u_{3}} \cdot \partial_{u_{1}} \partial_{u_{2}} \cdot \partial_{x_{31}}+\partial_{u_{1}} \cdot \partial_{u_{2}} \partial_{u_{3}} \cdot \partial_{x_{12}}
\end{aligned}
$$

Finally, the consistent cubic interactions are encoded in an arbitrary function $\mathcal{K}_{a_{1} a_{2} a_{3}}$ (2.16), and when expanded for different spins, they are expressed as

$$
\mathcal{K}_{a_{1} a_{2} a_{3}}=\sum_{s_{1}, s_{2}, s_{3}} \sum_{n=0}^{\min \left\{s_{1}, s_{2}, s_{3}\right\}} g_{a_{1} a_{2} a_{3}}^{s_{1} s_{2} s_{3}, n} \mathcal{G}^{n} \mathcal{Y}_{1}^{s_{1}-n} \mathcal{Y}_{2}^{s_{2}-n} \mathcal{Y}_{3}^{s_{3}-n}
$$

where the $g_{a_{1} a_{2} a_{3}, n}^{s_{1} s_{2} s_{3}, n}$, are coupling constants that might be fixed by the consistency of higher-order interactions. The number of derivatives of each vertex can be identified from the above expansion as

$$
s_{1}+s_{2}+s_{3}-2 n,
$$

so that the mass-dimensions of the coupling constants are

$$
\left[g_{a_{1} a_{2} a_{3}}^{s_{1} s_{2} s_{3}, n}\right]=\frac{6-d}{2}-s_{1}-s_{2}-s_{3}+2 n .
$$

Moreover, from the symmetry properties (2.9), the coupling constants inherit the symmetries

$$
g_{a_{2} a_{1} a_{3}}^{s_{2} s_{1} s_{3}, n}=g_{a_{1} a_{3} a_{2}}^{s_{1} s_{3} s_{2}, n}=(-1)^{s_{1}+s_{2}+s_{3}} g_{a_{1} a_{2} a_{3}}^{s_{1} s_{2} s_{3}, n} .
$$

As a result, the uncolored case is consistent only when the total spin $s_{1}+s_{2}+s_{3}$ is even.

\section{Free (A)dS HS gauge fields and Ambient-space formalism}

The full expressions for flat-space cubic vertices can be highly involved, and we have seen that the use of the TT constraints considerably simplifies the problem. Therefore, we employ the same strategy for the construction of cubic interactions in (A)dS, considering the system of (A)dS HS gauge fields subject to the TT constraints, where the transverse constraint is now with respect to the $(\mathrm{A}) \mathrm{dS}$ covariant derivative. Again, we expect that these constraints can be systematically foregone as in the flat-space case.

However, even after imposing the TT constraints, the (A)dS cubic-interaction problem continues to present non-trivial difficulties due to the non-commutativity of the covariant derivatives. We simplify the problem one step further making use of the ambient-space formalism of (A)dS fields.

In this section, we will concentrate on the free theory preparing the ground for the construction of cubic vertices which will be the subject of the next section. 


\subsection{Traceless and Transverse HS gauge fields}

To begin with, let us introduce the generating functions for symmetric tensor fields $\varphi_{\mu_{1} \cdots \mu_{s}}$ in $(\mathrm{A}) \mathrm{dS}$ as

$$
\varphi(x, u)=\sum_{s=0}^{\infty} \frac{1}{s !} \varphi_{\mu_{1} \cdots \mu_{s}}(x) u \cdot e^{\mu_{1}}(x) \cdots u \cdot e^{\mu_{s}}(x),
$$

where the fields are contracted with flat auxiliary variables $u^{\alpha}$ via the inverse vielbein $e_{\alpha}^{\mu}(x): u \cdot e^{\mu}(x)=u^{\alpha} e_{\alpha}^{\mu}(x)$. We consider, as in the flat-space case, the system of HS gauge fields with traceless and transverse constraints :

$$
\partial_{u}^{2} \varphi=0, \quad \partial_{u} \cdot e^{\mu} D_{\mu} \varphi=0,
$$

where now the transversality condition is to be considered with respect to the covariant derivative $D_{\mu}$, given by

$$
D_{\mu}:=\nabla_{\mu}+\frac{1}{2} \omega_{\mu}^{\alpha \beta}(x) u_{[\alpha} \partial_{u^{\beta]}} .
$$

Here the usual (A)dS-covariant derivative $\nabla_{\mu}$ acts on the tensor indices and $\omega_{\mu}^{\alpha \beta}$ is the (A)dS spin connection. With this definition of $D_{\mu}$, involving $\nabla_{\mu}$ instead of $\partial_{\mu}$, the (A)dS Laplacian operator is simply given by $D^{2}$. The quadratic action of HS gauge fields in (A)dS can be then simplified by the TT constraints to

$$
S_{\Lambda}^{(2)}=\frac{1}{2} \int d^{d} x \sqrt{-g}\left[\delta_{a_{1} a_{2}} e^{\partial_{u_{1}} \cdot \partial_{u_{2}}} \varphi^{a_{1}}\left(x_{1}, u_{1}\right)\left(D_{2}^{2}+\Lambda \mu_{2}\right) \varphi^{a_{2}}\left(x_{2}, u_{2}\right)\right]_{\substack{x_{i}=x \\ u_{i}=0}},
$$

where $g_{\mu \nu}$ is the (A)dS metric, and $\mu$ is the mass-squared-like operator:

$$
\mu:=\left(u \cdot \partial_{u}-2\right)\left(u \cdot \partial_{u}+d-4\right)-2,
$$

while $\Lambda$ is proportional to the cosmological constant, and the subscripts $i$ of $D_{i \mu}$ and $\mu_{i}$ indicate that the corresponding operators are acting on $\left(x_{i}, u_{i}\right)$. This action is invariant under the linearized HS gauge transformations:

$$
\delta_{\varepsilon}^{(0)} \varphi=u \cdot e^{\mu} D_{\mu} \varepsilon,
$$

with constrained gauge parameters satisfying the TT constraints:

$$
\partial_{u}^{2} \varepsilon=0, \quad \partial_{u} \cdot e^{\mu} D_{\mu} \varepsilon=0,
$$

as well as the field-equation-like differential constraint:

$$
\left[D^{2}+\Lambda u \cdot \partial_{u}\left(u \cdot \partial_{u}+d-2\right)\right] \varepsilon=0 .
$$

One could attempt to construct the cubic interactions at this stage as we did in the flatspace case, but as anticipated, the non-commutative nature of the covariant derivatives makes the problem involved. To circumvent this problem we choose to work in the ambient space approach expressing intrinsic (A)dS quantities in term of commutative flat-space ones. 


\subsection{Ambient space formalism}

In this subsection we review the ambient-space description for (A)dS HS gauge fields.

Ambient space formalism for $\mathbf{d S}$ tensors It is well known that one can embed the $d$-dimensional $\mathrm{dS}$ space in the $(d+1)$-dimensional flat space with metric:

$$
d s_{\mathrm{Amb}}^{2}=\eta_{M N} d X^{M} d X^{N}, \quad \eta=(-,+, \cdots,+) .
$$

The dS space is then defined as the hyper-surface $X^{2}=1 / \Lambda$ with $\Lambda>0$. The AdS space can be obtained from the dS space by an analytic continuation of $\Lambda,{ }^{15}$ and from now on we consider only the dS case with radius $L=1 / \sqrt{\Lambda}$.

We concentrate on the region of the ambient space with $X^{2}>0$, and consider the generating function of symmetric tensor fields $\Phi_{M_{1} \cdots M_{s}}$ given by

$$
\Phi(X, U)=\sum_{s=0}^{\infty} \frac{1}{s !} \Phi_{M_{1} \cdots M_{s}}(X) U^{M_{1}} \cdots U^{M_{s}} .
$$

These fields are equivalent to symmetric tensor fields in dS if they are homogeneous in $X^{M}$ and tangent to constant $X^{2}$ surfaces. The latter conditions translate at the level of the generating function into the following differential constraints:

$$
\left(X \cdot \partial_{X}-\Delta_{h}\right) \Phi=0, \quad X \cdot \partial_{U} \Phi=0,
$$

where $\Delta_{h}$ is the degree of homogeneity which will be fixed later in order to incorporate the gauge symmetries.

In order to identify ambient-space fields with dS fields, it is convenient to first parameterize the $X^{2}>0$ region with the radial coordinates $(R, x)$ given by

$$
X^{M}=R \hat{X}^{M}(x), \quad \hat{X}^{2}(x)=1,
$$

where $R=\sqrt{X^{2}}$ is the radius of $\mathrm{dS}$ and the $x^{\mu}$ 's are angular variables which also play the role of dS intrinsic coordinates. The metric in the radial coordinate system is then given by

$$
d s_{\mathrm{Amb}}^{2}=d R^{2}+\frac{R^{2}}{L^{2}} g_{\mu \nu}(x) d x^{\mu} d x^{\nu}=\tilde{e}^{R} \tilde{e}^{R}+\eta_{\alpha \beta} \tilde{e}^{\alpha} \tilde{e}^{\beta},
$$

where $g_{\mu \nu}(x)$ is the $\mathrm{dS}$ metric, and $\left(\tilde{e}^{R}, \tilde{e}^{\alpha}\right)$ is the ambient-space vielbein:

$$
\tilde{e}_{R}^{R}(R, x)=1, \quad \tilde{e}_{\mu}^{R}(R, x)=0, \quad \tilde{e}_{R}^{\alpha}(R, x)=0, \quad \tilde{e}_{\mu}^{\alpha}(R, x)=\frac{R}{L} e^{\alpha}{ }_{\mu}(x) .
$$

For the radial reduction of the generating function of HS fields, it is also necessary to perform for the auxiliary $U^{M}$-variables the change of coordinates

$$
U^{M}=\tilde{e}_{R}{ }^{M}(R, x) v+\tilde{e}_{\alpha}{ }^{M}(R, x) u^{\alpha}=\hat{X}^{M}(x) v+L \frac{\partial \hat{X}^{M}}{\partial x^{\mu}}(x) e_{\alpha}^{\mu}(x) u^{\alpha} .
$$

\footnotetext{
15 One can express AdS tensors in terms of ambient-space ones with a different signature, but the isomorphism between AdS and ambient-space gauge symmetries is not automatically realized, contrary to the dS case.
} 
With this change of variables from $(X, U)$ to $(R, x ; v, u)$, the homogeneous and tangent condition (3.11) are solved by the dS generating functions

$$
\Phi(R, x ; v, u)=\left(\frac{R}{L}\right)^{\Delta_{h}} \varphi(x, u),
$$

and it is useful for later use to present the ambient-space derivatives expressed in the radial coordinates:

$$
\begin{aligned}
& \partial_{X^{M}}=\hat{X}_{M} \partial_{R}+\frac{L^{2}}{R} \frac{\partial \hat{X}_{M}}{\partial x_{\mu}}\left[D_{\mu}+\frac{1}{L}\left(u \cdot e_{\mu} \partial_{v}-v \partial_{u} \cdot e_{\mu}\right)\right], \\
& \partial_{U^{M}}=\hat{X}_{M} \partial_{v}+L \frac{\partial \hat{X}_{M}}{\partial x_{\mu}} \partial_{u} \cdot e_{\mu} .
\end{aligned}
$$

Here $D_{\mu}$ is the dS covariant derivative defined in (3.3).

Gauge symmetries, Field equations and Actions So far the ambient-space tensor fields were not necessarily gauge fields. Now we want to address the case where the ambientspace fields admit linearized gauge symmetries of the form:

$$
\delta_{E}^{(0)} \Phi=U \cdot \partial_{X} E
$$

where $E(X, U)$ are generating functions of gauge parameters which are homogeneous and tangent according to eq. (3.11). From the expression of the gradient operator in radial coordinates:

$$
U \cdot \partial_{X}=v \partial_{R}+\frac{L}{R}\left[u \cdot e^{\mu} D_{\mu}+\frac{1}{L}\left(u^{2} \partial_{v}-v u \cdot \partial_{u}\right)\right],
$$

one can see that choosing the degrees of homogeneity for gauge fields and gauge parameters as

$$
\Phi(R, x ; v, u)=\left(\frac{R}{L}\right)^{u \cdot \partial_{u}-2} \varphi(x, u), \quad E(R, x ; v, u)=\left(\frac{R}{L}\right)^{u \cdot \partial_{u}} \varepsilon(x, u),
$$

the ambient-space gauge transformations (3.18) induce the dS ones in eq. (3.6). ${ }^{16}$ Moreover, the TT constraints imposed on the ambient-space gauge fields and gauge parameters are equivalent to the TT constraints on the dS ones. Most importantly, the field equations of the ambient-space HS gauge fields also induce the field equations of the dS fields, given by

$$
\partial_{X}^{2} \Phi=\left(\frac{R}{L}\right)^{u \cdot \partial_{u}-4}\left(D^{2}+\frac{1}{L^{2}} \mu\right) \varphi \approx 0 .
$$

Hence, the dS action (3.4) can be expressed in the ambient-space language as

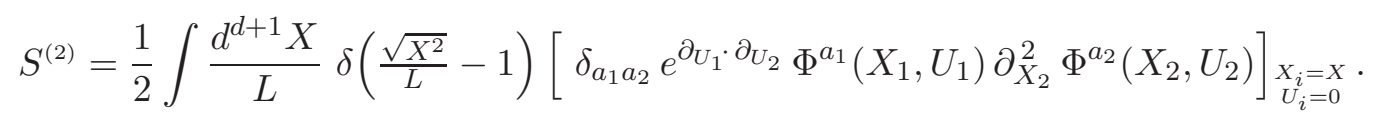

This is because the ambient-space integral splits into the dS one together with an additional radial integral as

$$
\int \frac{d^{d+1} X}{L}=\int_{0}^{\infty} \frac{d R}{L}\left(\frac{R}{L}\right)^{d} \int_{\mathrm{dS}} d^{d} x \sqrt{-g}
$$

\footnotetext{
${ }^{16}$ It was shown in [72] that different degrees of homogeneity give the gauge transformations of partially massless fields.
} 
while the ambient-space contractions simply reduce to the dS contractions:

$$
\left.\partial_{U_{1}} \cdot \partial_{U_{2}}(\cdots)\right|_{X_{i}=X}=\left.\left(\partial_{v_{1}} \partial_{v_{2}}+\partial_{u_{1}} \cdot \partial_{u_{2}}\right)(\cdots)\right|_{X_{i}=X},
$$

where of course $\partial_{v_{1}} \partial_{v_{2}}$ does not play a role whenever the contraction acts on fields $\varphi$ that do not depend on $v$.

Finally, we want to mention that there are two ways of formulating the dS action in the ambient-space formalism: one with the insertion of the $\delta$-function and the other without it. In this paper we choose the former in order to avoid ambiguities which might arise from the formally diverging radial integrals.

\section{Cubic interactions of HS gauge fields in (A)dS backgrounds}

In this section we construct the consistent cubic interactions of HS gauge fields in (A)dS within the TT setting. The interaction vertices will be provided in the ambient-space formalism, and we shall then show how to obtain the (A)dS-intrinsic expressions by radially reducing the ambient-space ones. We notice also that some key properties of the cubic vertices can be extracted from the ambient-space forms without re-expressing them in terms of intrinsic (A)dS quantities.

\subsection{Construction of consistent cubic interactions}

Our construction of the consistent cubic interactions in (A)dS is based on the ambientspace formalism, and let us notice that by considering all possible forms of ambient-space cubic vertices, one does not loose any generality since any dS vertex can be written as an ambient-space one making use of the following projector ${ }^{17}$ :

$$
P^{M N}=\frac{X^{M} X^{N}-\eta^{M N} X^{2}}{X^{2}} .
$$

However, as is suggested by the projector itself, the possible ambient-space cubic vertices are a bit more general than the flat-space ones. This can be also understood as a result of the break down of translational symmetries at the level of the ambient space induced by the $\delta$-function insertion. Therefore, the most general expression of the cubic interactions acquires one more argument, $X^{M}$, compared to the flat-space case (2.8):

$$
\begin{aligned}
S^{(3)}=\frac{1}{3 !} \int \frac{d^{d+1} X}{L} \delta\left(\frac{\sqrt{X^{2}}}{L}-1\right) & C_{a_{1} a_{2} a_{3}}\left(X ; \partial_{X_{1}}, \partial_{X_{2}}, \partial_{X_{3}} ; \partial_{U_{1}}, \partial_{U_{2}}, \partial_{U_{3}}\right) \times \\
& \times\left.\Phi^{a_{1}}\left(X_{1}, U_{1}\right) \Phi^{a_{2}}\left(X_{2}, U_{2}\right) \Phi^{a_{3}}\left(X_{3}, U_{3}\right)\right|_{\substack{X_{i}=X \\
U_{i}=0}}
\end{aligned}
$$

Since the arguments of $C_{a_{1} a_{2} a_{3}}$ do not commute, we decide to choose the ordering where all $X^{M}$ 's are placed on the left side of $\partial_{X_{i}}$ 's and $\partial_{U_{j}}$ 's .

As in the flat-space case, we can simplify the ansatz making use of all possible Lorentz invariants. However, compared to the flat case we have a few more quantities $X^{2}, X \cdot \partial_{X_{i}}$

17 See e.g. [57] for more details. 
and $X \cdot \partial_{U_{i}}$ and they should be placed on the left side of other Lorentz invariants in our conventions. In the following, we show that the dependence on these additional Lorentz invariant objects in the ansatz can be in fact neglected.

- First, $X^{2}$ simply becomes $L^{2}$ after the radial integration, and can be absorbed into the definition of $C_{a_{1} a_{2} a_{3}}$.

- Second, $X \cdot \partial_{X_{i}}$ is equivalent to $X_{i} \cdot \partial_{X_{i}}$ which essentially counts the number of $X_{i}$ 's and so can be absorbed into $C_{a_{1} a_{2} a_{3}}$ as well.

- Finally, $X \cdot \partial_{U_{i}}$ is equivalent to $X_{i} \cdot \partial_{U_{i}}$ which is nothing but the tangent condition (3.11). Hence, when it acts directly on fields it vanishes while acting on the derivatives produces

$$
\begin{aligned}
& \left(X \cdot \partial_{U}\right) \partial_{X^{M_{1}}} \cdots \partial_{X^{M_{n}}} \Phi \\
& \quad=-\sum_{m=1}^{n} \partial_{X^{M_{1}}} \cdots \partial_{X^{M_{m-1}}} \partial_{U^{M_{m}}} \partial_{X^{M_{m+1}}} \cdots \partial_{X^{M_{m-1}}} \Phi
\end{aligned}
$$

so that $X \cdot \partial_{U_{i}}$ is equivalent to a linear combination of the other Lorentz invariants.

After excluding these $X^{M}$-depending Lorentz invariants, one ends up with the same ansatz (2.8) which was the starting point of the flat-space construction. However, there is one other difference: total-derivative terms do not simply vanish due to the presence of the $\delta$-function insertion, and they do contribute to the action. To properly analyze the role of the total derivatives, the latter is denoted by $\partial_{X^{M}}=\partial_{X_{1}^{M}}+\partial_{X_{2}^{M}}+\partial_{X_{3}^{M}}$ and the other non-total derivatives by $\partial_{X_{i j}^{M}}:=\partial_{X_{i}^{M}}-\partial_{X_{j}^{M}}$. Then the ansatz for the cubic interactions is given by

$$
C_{a_{1} a_{2} a_{3}}\left(\partial_{X} ; \partial_{X_{12}}, \partial_{X_{23}}, \partial_{X_{31}} ; \partial_{U_{1}}, \partial_{U_{2}}, \partial_{U_{3}}\right)
$$

and this can be expanded in the powers of the total derivatives. By noticing that the total derivatives can be integrated by parts as

$$
\delta^{(n)}\left(\frac{\sqrt{X^{2}}}{L}-1\right) \partial_{X^{M}}=-\frac{1}{L^{2}} \delta^{(n+1)}\left(\frac{\sqrt{X^{2}}}{L}-1\right) X^{M},
$$

where $\delta^{(n)}(x):=\left(\frac{d}{d x}\right)^{n} \delta(x)$, and that the $X^{M}$-dependence in the vertex can be eliminated as we argued before, the general ansatz (4.4) for the cubic vertices can be recast in the form

$$
\delta\left(\frac{\sqrt{X^{2}}}{L}-1\right) C_{a_{1} a_{2} a_{3}}\left(\partial_{X} ; \partial_{X_{i j}}, \partial_{U_{i}}\right)=\sum_{n=0}^{\infty} \frac{1}{L^{n}} \delta^{(n)}\left(\frac{\sqrt{X^{2}}}{L}-1\right) C_{a_{1} a_{2} a_{3}}^{(n)}\left(\partial_{X_{i j}}, \partial_{U_{i}}\right) .
$$

Similarly to the flat-space construction, $C_{a_{1} a_{2} a_{3}}^{(n)}$ can be written as a function of the Lorentz invariants $\mathcal{Y}_{i}$ 's and $\mathcal{Z}_{i}$ 's, which are now the ambient-space analogues of (2.11):

$$
C_{a_{1} a_{2} a_{3}}^{(n)}\left(\partial_{X_{i j}}, \partial_{U_{i}}\right)=C_{a_{1} a_{2} a_{3}}^{(n)}\left(\mathcal{Y}_{i}, \mathcal{Z}_{i}\right)
$$


Making use of eq. (4.6), the gauge consistency condition reads

$$
\sum_{n=0}^{\infty} \frac{1}{L^{n}} \delta^{(n)}\left(\frac{\sqrt{X^{2}}}{L}-1\right)\left[C_{a_{1} a_{2} a_{3}}^{(n)}\left(\mathcal{Y}_{i}, \mathcal{Z}_{i}\right), U_{j} \cdot \partial_{X_{j}}\right] \approx 0
$$

where $\approx$ means equivalence modulo the free field equations $\partial_{X_{i}}^{2} \approx 0$. Moreover, the commutator in eq. (4.8) can be simplified making use of the identities:

$$
\left[\mathcal{Y}_{i}, U_{j} \cdot \partial_{X_{j}}\right] \approx \frac{1}{2} \delta_{i j} \epsilon_{i k \ell} \partial_{X} \cdot \partial_{X_{k \ell}}, \quad\left[\mathcal{Z}_{i}, U_{j} \cdot \partial_{X_{j}}\right]=-\frac{1}{2} \epsilon_{i j k} \mathcal{Y}_{k}+\frac{1}{2}\left|\epsilon_{i j k}\right| \partial_{X} \cdot \partial_{U_{k}}
$$

where the total derivatives terms play a role compared to the flat-space case (2.14) since they contribute to the $\delta^{(n+1)}$-order after an integration by parts. Taking into account the other commutator identities:

$$
\begin{aligned}
& {\left[X_{1} \cdot \partial_{U_{1}}, F\left(\mathcal{Y}_{i}, \mathcal{Z}_{i}\right)\right]=\left(\mathcal{Z}_{3} \partial_{\mathcal{Y}_{2}}-\mathcal{Z}_{2} \partial_{\mathcal{Y}_{3}}\right) F\left(\mathcal{Y}_{i}, \mathcal{Z}_{i}\right)} \\
& {\left[X_{1} \cdot \partial_{X_{1}}, F\left(\mathcal{Y}_{i}, \mathcal{Z}_{i}\right)\right]=-\frac{1}{2}\left[\left(\mathcal{Y}_{2}-\partial_{X} \cdot \partial_{U_{2}}\right) \partial_{\mathcal{Y}_{2}}+\left(\mathcal{Y}_{3}+\partial_{X} \cdot \partial_{U_{3}}\right) \partial \mathcal{Y}_{3}\right] F\left(\mathcal{Y}_{i}, \mathcal{Z}_{i}\right)} \\
& {\left[F\left(\mathcal{Y}_{i}, \mathcal{Z}_{i}\right), U_{1} \cdot \partial_{U_{1}}\right]=\left(\mathcal{Y}_{1} \partial_{\mathcal{Y}_{1}}+\mathcal{Z}_{2} \partial_{\mathcal{Z}_{2}}+\mathcal{Z}_{3} \partial_{\mathcal{Z}_{3}}\right) F\left(\mathcal{Y}_{i}, \mathcal{Z}_{i}\right)}
\end{aligned}
$$

we finally get the following differential equation and its cyclic permutations on (1)(2)(3) :

$$
\begin{aligned}
& \left(\mathcal{Y}_{1} \partial_{\mathcal{Z}_{2}}-\mathcal{Y}_{2} \partial_{\mathcal{Z}_{1}}\right) C_{a_{1} a_{2} a_{3}}^{(n)} \\
& +\frac{1}{L}\left[3\left(\mathcal{Y}_{1} \partial_{\mathcal{Y}_{1}}-\mathcal{Y}_{2} \partial_{\mathcal{Y}_{2}}\right) \partial_{\mathcal{Y}_{3}}-2 \mathcal{Z}_{3}\left(\partial_{\mathcal{Y}_{1}} \partial_{\mathcal{Z}_{1}}-\partial_{\mathcal{Y}_{2}} \partial_{\mathcal{Z}_{2}}\right)\right] C_{a_{1} a_{2} a_{3}}^{(n-1)} \\
& -\frac{1}{L^{2}} 3\left(\mathcal{Z}_{1} \partial_{\mathcal{Y}_{2}}-\mathcal{Z}_{2} \partial_{\mathcal{Y}_{1}}\right) \partial_{\mathcal{Y}_{3}}^{2} C_{a_{1} a_{2} a_{3}}^{(n-2)}=0 .
\end{aligned}
$$

Here $C_{a_{1} a_{2} a_{3}}^{(n)}$ vanishes for a negative $n$, and in the $L \rightarrow \infty$ limit these equations reduce to the flat-space condition (2.15).

Finally, the consistent parity-invariant cubic interactions in $(A) d S_{d}$ for $d \geq 4$ can be obtained as solutions of the differential recurrence equations (4.11), which can be solved from $n=0$ iteratively. For $n=0$ the corresponding equation coincides with the flat-space one (2.15), and its solutions are given again by arbitrary functions $\mathcal{K}_{a_{1} a_{2} a_{3}}$ as

$$
C_{a_{1} a_{2} a_{3}}^{(0)}\left(\mathcal{Y}_{i}, \mathcal{Z}_{i}\right)=\mathcal{K}_{a_{1} a_{2} a_{3}}\left(\mathcal{Y}_{i}, \mathcal{G}\right)
$$

where $\mathcal{G}:=\mathcal{Y}_{i} \mathcal{Z}_{i}$. On the contrary, for each $n \geq 1$ it is an inhomogeneous differential equation whose solutions are fixed up to a solution of the corresponding homogeneous equation. Each of these homogeneous solutions $\mathcal{K}_{a_{1} a_{2} a_{3}}^{\prime}\left(\mathcal{Y}_{i}, \mathcal{G}\right)$ induces a tail of particular solutions for higher orders, and provides additional solutions of eqs. (4.11):

$$
C_{a_{1} a_{2} a_{3}}=\frac{1}{L^{n}} \delta^{(n)}\left(\frac{\sqrt{X^{2}}}{L}-1\right) \mathcal{K}_{a_{1} a_{2} a_{3}}^{\prime}+\sum_{m=n+1}^{\infty} \frac{1}{L^{m}} \delta^{(m)}\left(\frac{\sqrt{X^{2}}}{L}-1\right) C_{a_{1} a_{2} a_{3}}^{(m)} .
$$

This observation seems to imply the presence of ambiguities in the (A)dS interactions, but these additional solutions are in fact redundancies. This is because, after the radial integration, different $\delta^{(n)}$ 's give just different spin-dependent constant factors. Therefore, any solution of the type (4.13) can be re-expressed in the form:

$$
C_{a_{1} a_{2} a_{3}}=\delta\left(\frac{\sqrt{X^{2}}}{L}-1\right) \tilde{\mathcal{K}}_{a_{1} a_{2} a_{3}}^{\prime}+\sum_{n=1}^{\infty} \frac{1}{L^{n}} \delta^{(n)}\left(\frac{\sqrt{X^{2}}}{L}-1\right) \tilde{C}_{a_{1} a_{2} a_{3}}^{(n)},
$$


where $\tilde{\mathcal{K}}_{a_{1} a_{2} a_{3}}^{\prime}$ and $\tilde{C}_{a_{1} a_{2} a_{3}}^{(n)}$ are proportional to $\mathcal{K}_{a_{1} a_{2} a_{3}}^{\prime}$ and $C_{a_{1} a_{2} a_{3}}^{(n)}$ with some spin-dependent factors. To reiterate, the aforementioned ambiguity can be recast into a redefinition of the original $C_{a_{1} a_{2} a_{3}}^{(0)}=\mathcal{K}_{a_{1} a_{2} a_{3}}$. Hence, a consistent cubic interaction is univocally determined from the choice of $C_{a_{1} a_{2} a_{3}}^{(0)}$.

\subsection{General solutions for consistent cubic interactions}

So far, we have shown that the consistent cubic interactions in (A)dS can be obtained solving the differential equations (4.11). The $\delta^{(0)}$-order solution was already obtained in terms of an arbitrary function $\mathcal{K}_{a_{1} a_{2} a_{3}}$, and what is left is to determine a particular solution for the higher order parts $C_{a_{1} a_{2} a_{3}}^{(n \geq 1)}$, keeping in mind that the ambiguities in the latter are redundancies.

In the following, we construct at once the full cubic vertex $C_{a_{1} a_{2} a_{3}}\left(\partial_{X} ; \partial_{X_{i j}}, \partial_{U_{i}}\right)$, comprising the full higher order tail of the $C_{a_{1} a_{2} a_{3}}^{(n)}$ 's, by making use of the following ansatz:

$$
C_{a_{1} a_{2} a_{3}}\left(\partial_{X} ; \partial_{X_{i j}}, \partial_{U_{i}}\right)=\mathcal{K}_{a_{1} a_{2} a_{3}}\left(\mathcal{Y}_{i}^{\Lambda}, \mathcal{G}^{\Lambda}\right),
$$

where $\mathcal{Y}_{i}^{\Lambda}$ 's and $\mathcal{G}^{\Lambda}$ are given by

$$
\begin{aligned}
& \mathcal{Y}_{i}^{\Lambda}=\mathcal{Y}_{i}+\alpha_{i} \partial_{U_{i}} \cdot \partial_{X} \\
& \mathcal{G}^{\Lambda}=\left(\mathcal{Y}_{1}+\beta_{1} \partial_{U_{1}} \cdot \partial_{X}\right) \mathcal{Z}_{1}+\left(\mathcal{Y}_{2}+\beta_{2} \partial_{U_{2}} \cdot \partial_{X}\right) \mathcal{Z}_{2}+\left(\mathcal{Y}_{3}+\beta_{3} \partial_{U_{3}} \cdot \partial_{X}\right) \mathcal{Z}_{3} .
\end{aligned}
$$

Notice first that this ansatz is a highly restricted one, with only six constants, compared to the general setting with an arbitrary number of $C_{a_{1} a_{2} a_{3}}^{(n)}$ 's. Nonetheless, the motivation is straightforward: we attempt to keep the form of the generating function $\mathcal{K}_{a_{1} a_{2} a_{3}}$ fixed. Notice as well that, although (4.16) contains explicitly total derivatives, the highest-derivative part of the vertices built from (4.16) do not, ensuring its non-triviality.

In order to examine the ansatz (4.15), we compute the gauge variation of the latter exercising some care in treating total derivatives. We first provide our solutions, leaving the detailed computation for the last part of this subsection. Requiring that the gauge variation vanish modulo $\partial_{X_{i}}^{2} \approx 0$, one ends up with the conditions

$$
\begin{aligned}
& \left(\alpha_{1}+1\right)\left(\alpha_{2}-1\right)+4=0, \\
& \left(\alpha_{1}+1\right)\left(\beta_{2}+1\right)+\left(\alpha_{1}-1\right)\left(\beta_{3}-1\right)=0, \\
& \left(\beta_{1}+1\right)\left(\beta_{2}+1\right)+\left(\beta_{3}-1\right)\left(\beta_{1}+\beta_{2}\right)=0,
\end{aligned}
$$

on the constants $\alpha_{i}$ and $\beta_{i}$ appearing in the ansatz (4.15), together with their cyclic permutations on the subscripts $i$ of $\alpha_{i}$ and $\beta_{i}$. If a solution for these equations exists, eq. (4.15) provides the consistent cubic interactions. Actually, eqs. (4.17-4.19) admit solutions parameterized by two constants $\alpha$ and $\beta$ :

$$
\begin{array}{lll}
\alpha_{1}=\alpha, & \alpha_{2}=\frac{\alpha-3}{\alpha+1}, & \alpha_{3}=-\frac{\alpha+3}{\alpha-1}, \\
\beta_{1}=\beta, & \beta_{2}=2 \frac{\alpha-\beta}{\alpha+1}-1, & \beta_{3}=-2 \frac{\alpha-\beta}{\alpha-1}+1 .
\end{array}
$$

As we have argued in the previous section, different choices of $\alpha$ and $\beta$ (which give different solutions for $C_{a_{1} a_{2} a_{3}}^{(n \geq 1)}$ ) can be reabsorbed into the definition of the functions $\mathcal{K}_{a_{1} a_{2} a_{3}}$ so that one can work with a particular choice of $\alpha$ and $\beta$ without loss of generality. 
Finally, the general solution for the (A)dS cubic-interaction problem is given by an arbitrary function $\mathcal{K}_{a_{1} a_{2} a_{3}}$ (4.15) together with eq. (4.20), and when the latter is expanded for different spins, we obtain the expression in eq. (1.1) with the coupling constants $g_{a_{1} a_{2} a_{3}}^{s_{1} s_{2} s_{3}, n}$. One can easily verify that when $s_{1}=s_{2}=0$ our results coincide with the interaction vertices constructed in [57]. The coupling constants have the same mass-dimensions (2.20) and the same permutation symmetries (2.21) as the flat-space ones, while each vertex is now not homogeneous in the number of the (A)dS-covariant derivatives since the ambient-space derivative (3.17) is not. However, the maximum number of derivatives of the vertex associated with $g_{a_{1} a_{2} a_{3}}^{s_{1} s_{2} s_{3}, n}$ can be easily identified as $s_{1}+s_{2}+s_{3}-2 n$. In the next section we shall see in more detail how this non-homogeneity is related to the inverse power expansion in the cosmological constant exhibited by the Fradkin-Vasiliev vertices.

\section{Proof at the $\delta^{(1)}$ level}

Here we show that the gauge invariance of the ansatz (4.15) is equivalent to the conditions of eqs. (4.17-4.19). Since all vertices of different spins are independent, we consider without loss of generality the case where $\mathcal{K}_{a_{1} a_{2} a_{3}}^{\Lambda}$ is given by an exponential function:

$$
\mathcal{K}_{a_{1} a_{2} a_{3}}^{\Lambda}=k_{a_{1} a_{2} a_{3}} e^{L \mathcal{V}}
$$

where $L$ is again the radius of dS and $\mathcal{V}$ is the sum of the arguments of $\mathcal{K}_{a_{1} a_{2} a_{3}}$ in eq. (4.15):

$$
\begin{aligned}
\mathcal{V}:= & \partial_{U_{1}} \cdot\left(\partial_{X_{23}}+\alpha_{1} \partial_{X}\right)+\partial_{U_{2}} \cdot\left(\partial_{X_{31}}+\alpha_{2} \partial_{X}\right)+\partial_{U_{3}} \cdot\left(\partial_{X_{12}}+\alpha_{3} \partial_{X}\right) \\
& +\partial_{U_{2}} \cdot \partial_{U_{3}} \partial_{U_{1}} \cdot\left(\partial_{X_{23}}+\beta_{1} \partial_{X}\right)+\partial_{U_{3}} \cdot \partial_{U_{1}} \partial_{U_{2}} \cdot\left(\partial_{X_{31}}+\beta_{2} \partial_{X}\right) \\
& +\partial_{U_{1}} \cdot \partial_{U_{2}} \partial_{U_{3}} \cdot\left(\partial_{X_{12}}+\beta_{3} \partial_{X}\right) .
\end{aligned}
$$

It is now convenient to use the following compact notation for the cubic action:

$$
S^{(3)}=\frac{1}{3 !} \int \delta k e^{L \mathcal{V}} \Phi_{1} \Phi_{2} \Phi_{3} \mid,
$$

where we use as a shorthand notation $k$ and $\Phi_{i}$ in place of $k_{a_{1} a_{2} a_{3}}$ and $\Phi^{a_{i}}\left(X_{i}, U_{i}\right)$, and $\mid$ at the end of equation denotes the evaluation $X_{i}=X$ and $U_{i}=0$. Performing the gauge variation with respect to $\Phi_{1}$, one then gets

$$
\delta_{E_{1}}^{(0)} S^{(3)}=\frac{1}{3 !} \int \delta k\left[\mathcal{V}, U_{1} \cdot \partial_{X_{1}}\right] e^{L \mathcal{V}} E_{1} \Phi_{2} \Phi_{3} \mid,
$$

where the commutator $\left[\mathcal{V}, U_{1} \cdot \partial_{X_{1}}\right]$ is given by the total derivative terms:

$$
\begin{aligned}
& {\left[\mathcal{V}, U_{1} \cdot \partial_{X_{1}}\right] \approx \partial_{X} \cdot \partial_{X_{23}}\left(1+\partial_{U_{2}} \cdot \partial_{U_{3}}\right)+\partial_{X} \cdot \partial_{X_{1}}\left(\alpha_{1}+\beta_{1} \partial_{U_{2}} \cdot \partial_{U_{3}}\right)} \\
& \quad+\partial_{X} \cdot \partial_{U_{2}}\left(\frac{1}{2} \partial_{X_{12}} \cdot \partial_{U_{3}}+\beta_{2} \partial_{X_{1}} \cdot \partial_{U_{3}}\right)+\partial_{X} \cdot \partial_{U_{3}}\left(\frac{1}{2} \partial_{X_{31}} \cdot \partial_{U_{2}}+\beta_{3} \partial_{X_{1}} \cdot \partial_{U_{2}}\right)
\end{aligned}
$$

After integrations by parts, one ends up with terms proportional to $X_{i} \cdot \partial_{X_{i}}$ and $X_{i} \cdot \partial_{U_{i}}$ which are exactly the operators appearing in the homogeneous and tangent conditions (3.11). To make these operators act directly on the fields, one must move them to the right of $e^{L \mathcal{V}}$ using the commutators $\left[X_{i} \cdot \partial_{X_{i}}, \mathcal{V}\right]$ and $\left[X_{i} \cdot \partial_{U_{i}}, \mathcal{V}\right]$. As a result one gets

$$
\delta_{E_{1}}^{(0)} S^{(3)} \approx-\frac{1}{3 !} \int \delta^{(1)} k e^{L \mathcal{V}} \mathcal{A} E_{1} \Phi_{2} \Phi_{3} \mid
$$


with

$$
\begin{gathered}
\mathcal{A}=\left(1+\partial_{U_{2}} \cdot \partial_{U_{3}}\right)\left[\frac{1}{L}\left(X_{2} \cdot \partial_{X_{2}}-X_{3} \cdot \partial_{X_{3}}\right)-\left(\alpha_{1} \partial_{X_{23}}+\partial_{X}\right) \cdot \partial_{U_{1}}+\left(\alpha_{2}+1\right) \partial_{X_{3}} \cdot \partial_{U_{2}}\right. \\
-\left(\alpha_{3}-1\right) \partial_{X_{2}} \cdot \partial_{U_{3}}-\left(\beta_{1} \partial_{X_{23}}+\partial_{X}\right) \cdot \partial_{U_{1}} \partial_{U_{2}} \cdot \partial_{U_{3}} \\
\left.+\left(\beta_{2}+1\right) \partial_{X_{3}} \cdot \partial_{U_{2}} \partial_{U_{3}} \cdot \partial_{U_{1}}-\left(\beta_{3}-1\right) \partial_{X_{2}} \cdot \partial_{U_{3}} \partial_{U_{1}} \cdot \partial_{U_{2}}\right] \\
+\left(\alpha_{1}+\beta_{1} \partial_{U_{2}} \cdot \partial_{U_{3}}\right)\left[\frac{1}{L} X_{1} \cdot \partial_{X_{1}}-\left(\alpha_{2}-1\right) \partial_{X_{1}} \cdot \partial_{U_{2}}-\left(\alpha_{3}+1\right) \partial_{X_{1}} \cdot \partial_{U_{3}}\right. \\
\left.-\left(\beta_{2}-1\right) \partial_{X_{1}} \cdot \partial_{U_{2}} \partial_{U_{3}} \cdot \partial_{U_{1}}-\left(\beta_{3}+1\right) \partial_{X_{1}} \cdot \partial_{U_{3}} \partial_{U_{1}} \cdot \partial_{U_{2}}\right] \\
+\left(\frac{1}{2} \partial_{X_{12}} \cdot \partial_{U_{3}}+\beta_{2} \partial_{X_{1}} \cdot \partial_{U_{3}}\right)\left[\frac{1}{L} X_{2} \cdot \partial_{U_{2}}-\left(\alpha_{3}-1\right) \partial_{U_{2}} \cdot \partial_{U_{3}}-\left(\alpha_{1}+1\right) \partial_{U_{1}} \cdot \partial_{U_{2}}\right. \\
\left.-\left(\beta_{3}-1\right) \partial_{U_{2}} \cdot \partial_{U_{3}} \partial_{U_{1}} \cdot \partial_{U_{2}}-\left(\beta_{1}+1\right) \partial_{U_{1}} \cdot \partial_{U_{2}} \partial_{U_{2}} \cdot \partial_{U_{3}}\right] \\
+\left(\frac{1}{2} \partial_{X_{31}} \cdot \partial_{U_{2}}+\beta_{3} \partial_{X_{1}} \cdot \partial_{U_{2}}\right)\left[\frac{1}{L} X_{3} \cdot \partial_{U_{3}}-\left(\alpha_{1}-1\right) \partial_{U_{3}} \cdot \partial_{U_{1}}-\left(\alpha_{2}+1\right) \partial_{U_{2}} \cdot \partial_{U_{3}}\right. \\
\left.-\left(\beta_{1}-1\right) \partial_{U_{3}} \cdot \partial_{U_{1}} \partial_{U_{2}} \cdot \partial_{U_{3}}-\left(\beta_{2}+1\right) \partial_{U_{2}} \cdot \partial_{U_{3}} \partial_{U_{3}} \cdot \partial_{U_{1}}\right] .
\end{gathered}
$$

The resulting term $\mathcal{A}$ contains $X_{i} \cdot \partial_{X_{i}}$ and $X_{i} \cdot \partial_{U_{i}}$ as well as other terms coming from the commutation relations. Here $X_{i} \cdot \partial_{U_{i}}$ vanishes by the tangent condition, and the homogenous condition replaces $X_{1} \cdot \partial_{X_{1}}$ and $X_{2} \cdot \partial_{X_{2}}-X_{3} \cdot \partial_{X_{3}}$ respectively, with $U_{1} \cdot \partial_{U_{1}}$ and $U_{2} \cdot \partial_{U_{2}}-U_{3} \cdot \partial_{U_{3}}$. Since the last two depend on $U_{i}$, pushing them to the left of $e^{L \mathcal{V}}$, they vanish when evaluated at $U_{i}=0$ and the only remaining contributions are the commutators. Collecting all the resulting terms one finally ends up with

$$
\delta_{E_{1}}^{(0)} S^{(3)} \approx-\frac{1}{3 !} \int \delta^{(1)} k(\mathcal{B}+\mathcal{C}) e^{L \mathcal{V}} E_{1} \Phi_{2} \Phi_{3} \mid
$$

where we have separated terms into the non-total-derivative part $\mathcal{B}$ (which involves only $\partial_{X_{i j}^{M}}$ but not $\partial_{X^{M}}$ ) and the total-derivative part $\mathcal{C}$ (which contains $\partial_{X^{M}}$ ). If the gauge variation $\delta_{1} S^{(3)}$ vanishes, $\mathcal{B}$ should vanish as well since there is no way to compensate it. In order to simplify the discussion one can split $\mathcal{B}$ as $\mathcal{B}=\mathcal{B}_{1}+\mathcal{B}_{2}+\mathcal{B}_{3}$, where the $\mathcal{B}_{n}$ 's are of order $n$ in the Lorentz invariants and are given respectively by

$$
\begin{aligned}
\mathcal{B}_{1}= & \frac{1}{2}\left[\left(\alpha_{1}+1\right)\left(\alpha_{2}-1\right)+4\right] \partial_{X_{31}} \cdot \partial_{U_{2}}-\frac{1}{2}\left[\left(\alpha_{3}+1\right)\left(\alpha_{1}-1\right)+4\right] \partial_{X_{12}} \cdot \partial_{U_{3}}, \\
\mathcal{B}_{2}= & \frac{1}{2}\left[\left(\alpha_{1}+1\right)\left(\beta_{2}+1\right)+\left(\alpha_{1}-1\right)\left(\beta_{3}-1\right)\right]\left(\partial_{X_{31}} \cdot \partial_{U_{2}} \partial_{U_{3}} \cdot \partial_{U_{1}}-\partial_{X_{12}} \cdot \partial_{U_{3}} \partial_{U_{1}} \cdot \partial_{U_{2}}\right) \\
& +\frac{1}{2}\left[\left(\alpha_{2}-1\right)\left(\beta_{1}+1\right)+\left(\alpha_{2}+1\right)\left(\beta_{3}-1\right)+4\right] \partial_{X_{31}} \cdot \partial_{U_{2}} \partial_{U_{2}} \cdot \partial_{U_{3}} \\
& -\frac{1}{2}\left[\left(\alpha_{3}-1\right)\left(\beta_{2}+1\right)+\left(\alpha_{3}+1\right)\left(\beta_{1}-1\right)+4\right] \partial_{X_{12}} \cdot \partial_{U_{3}} \partial_{U_{1}} \cdot \partial_{U_{2}} \\
\mathcal{B}_{3}= & \partial_{U_{2}} \cdot \partial_{U_{3}}\left\{\frac{1}{2}\left[\left(\beta_{1}+1\right)\left(\beta_{2}+1\right)+\left(\beta_{3}-1\right)\left(\beta_{1}+\beta_{2}\right)\right] \partial_{X_{31}} \cdot \partial_{U_{2}} \partial_{U_{2}} \cdot \partial_{U_{3}}\right. \\
& \left.\quad-\frac{1}{2}\left[\left(\beta_{3}+1\right)\left(\beta_{1}+1\right)+\left(\beta_{2}-1\right)\left(\beta_{3}+\beta_{1}\right)\right] \partial_{X_{12}} \cdot \partial_{U_{3}} \partial_{U_{1}} \cdot \partial_{U_{2}}\right\}
\end{aligned}
$$

Since $\mathcal{B}_{n}$ 's are independent, each $\mathcal{B}_{n}$ should vanish separately. Moreover, since we have considered so far the gauge consistency only with respect to $\delta^{(0)} \Phi^{a_{1}}\left(X_{1}, U_{1}\right)$ we have still to take into account the gauge invariance with respect to $\delta^{(0)} \Phi^{a_{2}}\left(X_{2}, U_{2}\right)$ and $\delta^{(0)} \Phi^{a_{3}}\left(X_{3}, U_{3}\right)$. These give the same conditions just recovered here for $\delta^{(0)} \Phi^{a_{1}}\left(X_{1}, U_{1}\right)$ but with cyclic permutations on the subscripts $i$ of $\alpha_{i}$ and $\beta_{i}$. Finally, the equations $\mathcal{B}_{1}=0, \mathcal{B}_{2}=0$ and $\mathcal{B}_{3}=0$ give respectively the conditions $(4.17),(4.18)$ and (4.19) on the constants $\alpha_{i}$ and $\beta_{i}$. 
To complete the proof, one should also compute the total-derivative part $\mathcal{C}$ in (4.28) and verify whether it imposes additional constraints on the $\alpha_{i}$ and $\beta_{i}$. Actually, $\mathcal{C}$ is vanishing with the conditions (4.17-4.19), and hence the latter equations are sufficient. However, this cannot be seen simply at the present level $\delta^{(1)}$, but needs to be carefully analyzed at the next level $\delta^{(2)}$. The details of the proof can be found in the Appendix B.

\subsection{Reduction to (A)dS-intrinsic expressions}

The cubic vertices (1.1) constructed in the ambient-space formalism are given in terms of the Lorentz invariants $\partial_{U_{i}} \cdot \partial_{X_{j}}$ and $\partial_{U_{i}} \cdot \partial_{U_{j}}$, and the expressions are compact but implicit with respect to (A)dS. The explicit expressions in terms of (A)dS-intrinsic quantities can be obtained making use of the radial reduction formulas (3.17) and (3.20). A convenient way for the reduction is to express the Lorentz invariants in terms of the following (A)dSintrinsic bi-local quantities:

$$
\begin{aligned}
Z\left(x_{i}, x_{j}\right) & :=\hat{X}_{M}\left(x_{i}\right) \hat{X}^{M}\left(x_{j}\right), \\
H_{\mu}\left(x_{i}, x_{j}\right) & :=L \frac{\partial \hat{X}_{M}\left(x_{i}\right)}{\partial x_{i}^{\mu}} \hat{X}^{M}\left(x_{j}\right), \\
G_{\mu \nu}\left(x_{i}, x_{j}\right) & :=L^{2} \frac{\partial \hat{X}_{M}\left(x_{i}\right)}{\partial x_{i}^{\mu}} \frac{\partial \hat{X}^{M}\left(x_{j}\right)}{\partial x_{j}^{\nu}},
\end{aligned}
$$

whose coincident-point limits are given by

$$
Z(x, x)=1, \quad H^{\mu}(x, x)=0, \quad G^{\mu \nu}(x, x)=g^{\mu \nu}(x) .
$$

Here the indices of the bi-local quantities are raised or lowered with the local metric tensor. With these conventions, the ambient-space Lorentz invariant operators can be written as

$$
\begin{aligned}
\partial_{U_{i}} \cdot \partial_{X_{j}}= & {\left[\partial_{v_{i}} Z\left(x_{i}, x_{j}\right)+\partial_{u_{i}^{\mu}} H^{\mu}\left(x_{i}, x_{j}\right)\right] \partial_{R_{j}} } \\
& +\left[\partial_{v_{i}} H^{\nu}\left(x_{j}, x_{i}\right)+\partial_{u_{i}^{\mu}} G^{\mu \nu}\left(x_{i}, x_{j}\right)\right]\left[D_{j \nu}+\frac{1}{L}\left(u_{j \nu} \partial_{v_{j}}-v_{j} \partial_{u_{j}^{\nu}}\right)\right] \frac{L}{R_{j}}, \\
\partial_{U_{i}} \cdot \partial_{U_{j}}= & \partial_{v_{i}} Z\left(x_{i}, x_{j}\right) \partial_{v_{j}}+\partial_{u_{i}^{\mu}} H^{\mu}\left(x_{i}, x_{j}\right) \partial_{v_{j}}+\partial_{v_{i}} H^{\nu}\left(x_{j}, x_{i}\right) \partial_{u_{j}^{\nu}} \\
& +\partial_{u_{i}^{\mu}} G^{\mu \nu}\left(x_{i}, x_{j}\right) \partial_{u_{j}^{\nu}}
\end{aligned}
$$

where we have also introduced the compact notation

$$
u_{i}^{\mu}:=u_{i}^{\alpha} e_{\alpha}^{\mu}\left(x_{i}\right), \quad \partial_{u_{i}^{\mu}}:=\partial_{u_{i}^{\alpha}} e^{\alpha}{ }_{\mu}\left(x_{i}\right) .
$$

These quantities are more convenient than the flat auxiliary variables for the explicit computations since they commute with the (A)dS-covariant derivative:

$$
\left[D_{i \mu}, u_{j}^{\nu}\right]=0, \quad\left[D_{i \mu}, \partial_{u_{j}^{\nu}}\right]=0 .
$$

The advantage of the bi-local quantities (4.34) rests on the fact that they are closed under the action of the (A)dS-covariant derivatives, as one can see by explicit computation:

$$
\begin{array}{cl}
D_{i \mu} Z\left(x_{i}, x_{j}\right)=\frac{1}{L} H_{\mu}\left(x_{i}, x_{j}\right), & D_{i \nu} H_{\mu}\left(x_{i}, x_{j}\right)=-g_{\mu \nu}\left(x_{i}\right) \frac{1}{L} Z\left(x_{i}, x_{j}\right), \\
D_{j \nu} H_{\mu}\left(x_{i}, x_{j}\right)=\frac{1}{L} G_{\mu \nu}\left(x_{i}, x_{j}\right), & D_{i \rho} G_{\mu \nu}\left(x_{i}, x_{j}\right)=-g_{\rho \mu}\left(x_{i}\right) \frac{1}{L} H_{\nu}\left(x_{j}, x_{i}\right) .
\end{array}
$$


Therefore, the ambient-space cubic vertices (1.1) can be reduced to the (A)dS-intrinsic ones with some algebra. Notice that the ambient-space derivatives $\partial_{X_{i}^{M}}$ do not always reduce to the (A)dS covariant ones $D_{i \mu}$, but they can produce some powers of $1 / L^{2}$ either via the contractions between $\partial_{v_{i}} / L$ 's and $v_{i} / L$ 's or via the actions on bi-local quantities. Hence, an ambient-space vertex with a number $\Delta$ of ambient-space derivatives results in different $(\mathrm{A}) \mathrm{dS}$ vertices whose number of covariant derivatives varies within the range $\Delta, \Delta-2, \cdots, 1$ (or 0 ). Whenever the number of derivatives decreases by two, the corresponding mass-dimension is compensated by a factor $1 / L^{2}$.

\section{Example: 3-3-2 vertex with lowest number of derivatives}

Let us deal with an explicit example in order to see how this radial reduction works. We have chosen the 3-3-2 example with the least number of derivatives because it is both one of the simplest examples of HS interactions and one of the vertices constructed by Fradkin and Vasiliev in the frame-like formalism. The $3-3-2$ vertex was also obtained in $[50,51]$ in terms of metric-like fields.

For simplicity, we leave aside the Chan-Paton factors and choose $\alpha$ and $\beta$ in a way ${ }^{18}$ that the cubic action has a symmetric form:

$$
\begin{aligned}
& S^{(3)}=-\frac{2}{3} g^{332,2} \int \frac{d^{d+1} X}{L} \delta\left(\frac{\sqrt{X^{2}}}{L}-1\right)\left(\mathcal{G}^{\Lambda}\right)^{2} \partial_{U_{1}} \cdot \partial_{X_{2}} \partial_{U_{2}} \cdot \partial_{X_{1}} \times \\
& \times\left.\Phi^{(3)}\left(X_{1}, U_{1}\right) \Phi^{(3)}\left(X_{2}, U_{2}\right) \Phi^{(2)}\left(X_{3}, U_{3}\right)\right|_{\substack{x_{1}=X_{2}=X_{3}=X \\
U_{1}=U_{2}=U_{3}=0}}
\end{aligned}
$$

where $\mathcal{G}^{\Lambda}$ is given by

$$
\mathcal{G}^{\Lambda}=2\left[\partial_{U_{2}} \cdot \partial_{U_{3}} \partial_{U_{1}} \cdot \partial_{X_{2}}-\partial_{U_{1}} \cdot \partial_{U_{3}} \partial_{U_{2}} \cdot \partial_{X_{1}}+\frac{1}{2} \partial_{U_{1}} \cdot \partial_{U_{2}} \partial_{U_{3}} \cdot \partial_{X_{12}}\right] \cdot
$$

Expanding $\left(\mathcal{G}^{\Lambda}\right)^{2}$ gives rise to six terms, and in order to describe the procedure let us consider first the term

$$
\left(\partial_{U_{2}} \cdot \partial_{U_{3}}\right)^{2}\left(\partial_{U_{1}} \cdot \partial_{X_{2}}\right)^{3} \partial_{U_{2}} \cdot \partial_{X_{1}}
$$

Using (4.37) and (4.36), one gets

$$
\begin{aligned}
& {\left[\partial_{v_{2}} Z_{23} \partial_{v_{3}}+\partial_{u_{2}} \cdot H_{3}^{2} \partial_{v_{3}}+\partial_{u_{3}} \cdot H_{2}^{3} \partial_{v_{2}}+\partial_{u_{2}} \cdot G^{23} \cdot \partial_{u_{3}}\right]^{2}} \\
& \times\left[\left(\partial_{v_{1}} Z_{12}+\partial_{u_{1}} \cdot H^{1}{ }_{2}\right) \partial_{R_{2}}+\left(\partial_{v_{1}} H_{1}{ }^{2}+\partial_{u_{1}} \cdot G^{12}\right) \cdot\left[D_{2}+\frac{1}{L}\left(u_{2} \partial_{v_{2}}-v_{2} \partial_{u_{2}}\right)\right] \frac{L}{R_{2}}\right]^{3} \\
& \times\left[\left(\partial_{v_{2}} Z_{21}+\partial_{u_{2}} \cdot H^{2}{ }_{1}\right) \partial_{R_{1}}+\left(\partial_{v_{2}} H_{2}{ }^{1}+\partial_{u_{2}} \cdot G^{21}\right) \cdot\left[D_{1}+\frac{1}{L}\left(u_{1} \partial_{v_{1}}-v_{1} \partial_{u_{1}}\right)\right] \frac{L}{R_{1}}\right]
\end{aligned}
$$

where the subscripts or superscripts of $Z, H$ and $G$ encode the bi-local dependence on $\left(x_{i}, x_{j}\right)$, in particular,

$$
\left(H^{i}{ }_{j}\right)_{\mu}=H_{\mu}\left(x_{i}, x_{j}\right), \quad\left(H_{i}{ }^{j}\right)_{\mu}=H_{\mu}\left(x_{j}, x_{i}\right) .
$$

\footnotetext{
${ }^{18}$ We take the $\epsilon \rightarrow 0$ limit with $\alpha=1-2 \epsilon$ and $\beta=1+\epsilon$. Even though the second line's last factor in (1.1) diverges, it does not matter since we consider the case $n=s_{3}$.
} 
Even though eq. (4.44) has a rather complicated structure, many simplifications can be made. First, since the operator (4.43) is acting on

$$
\left.\frac{R_{1}}{L} \frac{R_{2}}{L} \varphi^{(3)}\left(x_{1}, u_{1}\right) \varphi^{(3)}\left(x_{2}, u_{2}\right) \varphi^{(2)}\left(x_{3}, u_{3}\right)\right|_{u_{i}=v_{i}=0},
$$

the dependence in $R_{i}$ and $v_{i}$ can be removed performing all possible contractions. Second, the coincident limit (4.35) simplifies some of the bi-local quantities, and the formula (4.44) becomes

$$
\begin{gathered}
\left(\partial_{u_{2}} \cdot \partial_{u_{3}}\right)^{2}\left[\left\{\left(\partial_{u_{1}} \cdot D_{2}\right)^{2}\left(\partial_{u_{1}} \cdot G^{12} \cdot D_{2}+\frac{1}{L} \partial_{u_{1}} \cdot H^{1}{ }_{2}\right)-\frac{3}{L^{2}} u_{2} \cdot \partial_{u_{1}} \partial_{u_{1}} \cdot \partial_{u_{2}} \partial_{u_{1}} \cdot D_{2}\right\} \times\right. \\
\quad \times\left(\partial_{u_{2}} \cdot G^{21} \cdot D_{1}+\frac{1}{L} \partial_{u_{2}} \cdot H^{2}{ }_{1}\right) \\
+\frac{1}{L}\left\{\frac{1}{L}\left(\partial_{u_{1}} \cdot D_{2}\right)^{2}-\partial_{u_{1}} \cdot D_{2} H_{1}{ }^{2} \cdot D_{2}\left(\partial_{u_{1}} \cdot G^{12} \cdot D_{2}+\frac{1}{L} \partial_{u_{1}} \cdot H^{1}{ }_{2}\right)\right. \\
\quad+\frac{1}{L^{2}} \partial_{u_{1}} \cdot D_{2} u_{2} \cdot H^{2}{ }_{1} \partial_{u_{1}} \cdot G^{12} \cdot \partial_{u_{2}}-\left(\partial_{u_{1}} \cdot D_{2}\right)^{2}\left(H_{1}{ }^{2} \cdot D_{2}+\frac{1}{L} Z_{12}\right) \\
\left.\left.\quad+\frac{2}{L^{2}} u_{2} \cdot \partial_{u_{1}} \partial_{u_{1}} \cdot D_{2} \partial_{u_{2}} \cdot H^{2}{ }_{1}\right\} \partial_{u_{1}} \cdot G^{12} \cdot \partial_{u_{2}}\right]
\end{gathered}
$$

Finally, the property (4.40) enables one to remove all bi-local quantities replacing them with some powers of $L$. At the end, one obtains the (A)dS intrinsic expression for the operator (4.43) as

$$
\left(\partial_{u_{2}} \cdot \partial_{u_{3}}\right)^{2}\left(\partial_{u_{1}} \cdot D_{2}\right)^{3} \partial_{u_{2}} \cdot D_{1}-\frac{6}{L^{2}} \partial_{u_{1}} \cdot \partial_{u_{2}} \partial_{u_{1}} \cdot \partial_{u_{3}} \partial_{u_{2}} \cdot \partial_{u_{3}} \partial_{u_{1}} \cdot D_{2} \partial_{u_{2}} \cdot D_{1} \cdot
$$

Notice that the first term has the same form of (4.43) with the replacement of $\left(\partial_{X_{i}}, \partial_{U_{i}}\right)$ by $\left(D_{i}, \partial_{u_{i}}\right)$, but the second term has a lower number of derivatives and is proportional to $1 / L^{2}$.

Five other terms in the expansion (4.41) can be computed in a similar way (see Appendix $\mathrm{C}$ for more details), and the cubic action (4.41) can be finally expressed solely in terms of $(\mathrm{A}) \mathrm{dS}$ intrinsic quantities as

$$
\begin{aligned}
& S^{(3)}=-\frac{8}{3} g^{332,2} \int d^{d} x \sqrt{-g} \times \\
& \times[ \\
& \quad\left[\left(\partial_{u_{2}} \cdot \partial_{u_{3}} \partial_{u_{1}} \cdot D_{2}-\partial_{u_{1}} \cdot \partial_{u_{3}} \partial_{u_{2}} \cdot D_{1}+\frac{1}{2} \partial_{u_{1}} \cdot \partial_{u_{2}} \partial_{u_{3}} \cdot D_{12}\right)^{2} \partial_{u_{1}} \cdot D_{2} \partial_{u_{2}} \cdot D_{1}\right. \\
& \quad+\frac{4}{L^{2}} \partial_{u_{1}} \cdot \partial_{u_{2}}\left[\left(\partial_{u_{2}} \cdot \partial_{u_{3}}\right)^{2}\left(\partial_{u_{1}} \cdot D_{2}\right)^{2}+\left(\partial_{u_{1}} \cdot \partial_{u_{3}}\right)^{2}\left(\partial_{u_{2}} \cdot D_{1}\right)^{2}-3 \partial_{u_{1}} \cdot \partial_{u_{3}} \partial_{u_{2}} \cdot \partial_{u_{3}} \partial_{u_{1}} \cdot D_{2} \partial_{u_{2}} \cdot D_{1}\right] \\
& \quad+\frac{3}{L^{2}}\left(\partial_{u_{1}} \cdot \partial_{u_{2}}\right)^{2}\left[\partial_{u_{2}} \cdot \partial_{u_{3}} \partial_{u_{1}} \cdot D_{2}-\partial_{u_{1}} \cdot \partial_{u_{3}} \partial_{u_{2}} \cdot D_{1}+\frac{1}{6} \partial_{u_{1}} \cdot \partial_{u_{2}} \partial_{u_{3}} \cdot D_{1_{2}}\right] \partial_{u_{3}} \cdot D_{12} \\
& \quad-\frac{5}{4 L^{2}}\left(\partial_{u_{1}} \cdot \partial_{u_{2}}\right)^{2}\left[\partial_{u_{2}} \cdot \partial_{u_{3}} \partial_{u_{1}} \cdot D_{2} \partial_{u_{3}} \cdot D_{1}+\partial_{u_{1}} \cdot \partial_{u_{3}} \partial_{u_{2}} \cdot D_{1} \partial_{u_{3}} \cdot D_{2}\right] \\
& \left.\quad-\frac{7 d+29}{2 L^{4}}\left(\partial_{u_{1}} \cdot \partial_{u_{2}}\right)^{2} \partial_{u_{1}} \cdot \partial_{u_{3}} \partial_{u_{2}} \cdot \partial_{u_{3}}\right]\left.\varphi^{(3)}\left(x_{1}, u_{1}\right) \varphi^{(3)}\left(x_{2}, u_{2}\right) \varphi^{(2)}\left(x_{3}, u_{3}\right)\right|_{\substack{x_{1}=x_{2}=x_{3}=x \\
u_{1}=u_{2}=u_{3}=0}}, \quad \text { (4.49) }
\end{aligned}
$$

where we organized the various contributions according to the number of (A)dS covariant derivatives.

\section{Discussion and Outlook}

In this paper, we have obtained the general solution of the cubic-interaction problem of HS gauge fields in (A)dS within the setting of the TT constraints that we have defined in the 
Introduction. Interestingly, the structure of the vertices, when expressed in the ambientspace formalism, coincides with the flat-space ones up to non-trivial total-derivative terms whose form is completely constrained by the gauge consistency.

\subsection{Relation to the Fradkin-Vasiliev vertices}

Let us consider the $s-s-2$ vertices, which were originally constructed by Fradkin and Vasiliev (FV). They correspond to the case $s_{1}=s_{2}=s$ and $s_{3}=n=2$ in (1.1), so that they are the vertices with lowest number of derivatives. With the same choice of $\alpha$ and $\beta$ as in (4.41), they are given by

$$
\begin{aligned}
S^{(3)}=g^{s s 2,2} \int \frac{d^{d+1} X}{L} \delta\left(\frac{\sqrt{X^{2}}}{L}-1\right)\left(\mathcal{G}^{\Lambda}\right)^{2}\left(\partial_{U_{1}} \cdot \partial_{X_{2}} \partial_{U_{2}} \cdot \partial_{X_{1}}\right)^{s-2} \times \\
\times \Phi^{(s)}\left(X_{1}, U_{1}\right) \Phi^{(s)}\left(X_{2}, U_{2}\right) \Phi^{(2)}\left(X_{3}, U_{3}\right) \begin{array}{c}
U_{1=X_{2}=X_{3}=X}, \\
U_{1}=U_{2}=U_{3}=0
\end{array}
\end{aligned}
$$

where, for simplicity, we have absorbed a numerical factor into the definition of the coupling constant. In the previous section, we have shown how to express ambient differential operators in terms of (A)dS-intrinsic quantities. Likewise, expressing the operators $\left(\partial_{U_{1}} \cdot \partial_{X_{2}} \partial_{U_{2}} \cdot \partial_{X_{1}}\right)^{s-2}$ in the above formula using (4.36) yields an expression in terms of (A)dS-covariant derivatives, bi-local quantities and also the $v_{i}$ 's. Taking the ordering where all (A)dS-covariant derivatives are placed on the RHS, one gets

$$
\left(\partial_{U_{1}} \cdot \partial_{X_{2}} \partial_{U_{2}} \cdot \partial_{X_{1}}\right)^{s-2}=\mathcal{A}_{s-2}+\Lambda \mathcal{A}_{s-3}+\cdots+\Lambda^{s-2} \mathcal{A}_{0}
$$

where $\mathcal{A}_{r}$ is the portion containing the $2 r$-th power of the (A)dS-covariant derivatives, or $\mathcal{A}_{r} \propto D^{2 r}$. Plugging (5.2) into (5.1), the $s-s-2$ vertex admits a similar expansion given by

$$
S^{(3)}=g^{s s 2,2}\left[A_{s}+\Lambda A_{s-1}+\cdots+\Lambda^{s-2} A_{2}\right]
$$

with

$$
A_{r+2}=\left.\int \frac{d^{d+1} X}{L} \delta\left(\frac{\sqrt{X^{2}}}{L}-1\right)\left(\mathcal{G}^{\Lambda}\right)^{2} \mathcal{A}_{r} \Phi^{(s)} \Phi^{(s)} \Phi^{(2)}\right|_{\substack{X_{1}=X_{2}=X_{3}=X \\ U_{1}=U_{2}=U_{3}=0}} .
$$

Notice that each $A_{r}$ is separately gauge invariant under the spin 2 gauge transformation, and this is due to the fact that the $\mathcal{A}_{r}$ 's trivially commute with the spin 2 gauge transformation. Notice as well that $A_{r}$ involves $2(r-2)$ or $2(r-1)(\mathrm{A}) \mathrm{dS}$ covariant derivatives, since the action of $\left(\mathcal{G}^{\Lambda}\right)^{2}$ may or may not add two additional derivatives.

This expansion of the vertex is quite similar to the one obtained by FV, and in fact one can make it as an expansion in inverse powers of $\Lambda$ by redefining the coupling constant and the fields as

$$
g^{s s 2,2}=\frac{\sqrt{G}}{\Lambda^{s-2}} \lambda_{s}, \quad \varphi^{(2)}=\frac{1}{\sqrt{G}} h, \quad \varphi^{(s)}=\frac{1}{\sqrt{G}} \phi^{(s)} .
$$

The coupling constant $g^{s s 2,2}$ has mass-dimension $(2-d) / 2-2(s-2)$, while with this redefinition the new coupling constant $\lambda_{s}$ together with the new fields have vanishing mass-dimension. Finally, the expansion (5.3) becomes

$$
S^{(3)}=\frac{\lambda_{s}}{G}\left[\tilde{A}_{2}+\frac{1}{\Lambda} \tilde{A}_{3}+\cdots+\frac{1}{\Lambda^{s-2}} \tilde{A}_{s}\right]
$$


where $\tilde{A}_{r}$ 's are given schematically by eq. (1.5) in terms of dimensionless fields $\phi^{(s)}$ and $h$.

Two remarks are in order. First, the lowest-derivative part $\tilde{A}_{2}$ of the above expression should involve the gravitational minimal coupling as well as non-minimal ones which do not deform the gauge transformation. Therefore, the simplest way to see this link is to analyze how the vertices constructed in this paper deform the gauge transformation and the gauge algebra. We leave this issue for future work. Second, the highest-derivative part (the so-called seed coupling, according to [77]) has the same form as the flat-space vertices with $\partial_{x_{i}^{\mu}}$ 's replaced by $D_{i \mu}$ 's. The relation between the gravitational minimal coupling and the seed coupling was already noticed in [51], and in the present work we can see how both lower-derivative and seed couplings come out at the same time from the ambient-space vertices. From a more general perspective, it would be interesting to investigate the relation between the present construction (in metric-like approach) and the recent frame-like one [33].

Boulanger-Leclercq-Sundell limit Since a curved space looks flat in the short-distance limit, the dominant term of curved-space actions in the limit should correspond to flatspace ones. One may expect to obtain in this way the flat-space vertices from the FV ones, but because of the inverse power expansion in $\Lambda$ the dominant terms diverge in the limit. In [51], the authors considered a particular limit of the FV system in order to extract flatspace information from AdS interactions. More precisely, they considered the limit where not only the cosmological constant but also the gravitational constant and fields scale as

$$
\begin{array}{cl}
\Lambda=\epsilon \tilde{\Lambda}, & G=\epsilon^{2(s-2)} \tilde{G}, \\
h=\epsilon^{s-2} \tilde{h}, & \phi^{(s)}=\epsilon^{s-2} \tilde{\phi}^{(s)},
\end{array}
$$

with $\epsilon \rightarrow 0$. Under this rescaling, the quadratic action remains invariant, but the cubic vertices scale in a way that only the seed coupling survives and one gets the flat-space vertices with $2 s-2$ derivatives.

In our setting, this can be understood at the level of (5.3), where the flat-space limit is not singular for fixed (or non-scaling) $g^{s s 2,2}$ and $\varphi^{(s)}$ 's, and the flat-space cubic vertices are recovered. In this respect, the rescaling (5.7) can be viewed as a particular flat-space limit in (5.5) which holds $g^{s s 2,2}$ and $\varphi^{(s)}$ 's finite.

\subsection{Outlook}

Let us conclude by summarizing our results and strategies of the present paper and by describing their possible applications from a more general perspective.

First, we observed that the flat-space interactions play a key role, through the ambientspace formalism, in understanding and controlling cubic interactions in any constant curvature background. Second, the simplified TT (or on-shell) system makes possible to identify the consistent cubic interactions and this observation has recently led to the proposal [48] for quartic and higher-order HS interactions by one of the authors. We expect as well that many other key properties of the interactions can be appreciated at this simpler level.

The aforementioned perspectives open a new window for a systematic analysis of many other aspects of HS theory. First of all, the issue of non-Abelian HS gauge algebras in (A)dS 
and flat space together with their relations might be addressed ${ }^{19}$. In particular, it is interesting to draw some more lessons on the HS geometry ${ }^{20}$ from the relations between the minimal (A)dS couplings and non-minimal ambient-space ones. Moreover, the nature of non-localities, which appear in the flat-space Lagrangian starting from the quartic order, can be clarified from this point of view: flat-space non-localities might fit within the Vasiliev's system with the aid of the ambient-space formalism. If so, the strategy employed in this work can give an additional motivation for the flat-space HS gauge theory.

Further interesting applications of our results can be found in massive HS field theories ${ }^{21}$ of which String Theory is the most important example. Actually, the interactions of massive HS fields can be investigated with techniques similar to those used in this paper, and we hope to address on this question soon. It is indeed believed by many authors ${ }^{22}$ that the masses of HS fields can play a role similar to that of the cosmological constant of massless HS theories, and the understanding of this relation can give more insights on the very nature of String Theory. ${ }^{23}$

Moreover, other applications can be found in the AdS/CFT correspondence, which has been applied to HS theories starting from $[92,93] .{ }^{24}$ We expect that the ambientspace representation of interacting vertices simplifies the computations of $n$-point functions. Moreover, loop computations might be addressed within this formalism, shedding some light on the quantum aspects of HS gauge theories. We hope to report on these issues in the near future.

\section{Acknowledgments}

We are grateful to X. Bekaert, N. Boulanger, A. Campoleoni, L. Lopez, J. Mourad, P. Sundell and M. Tsulaia for helpful conversations, and especially to A. Sagnotti for the reading of the manuscript and for many suggestions. The present research was supported in part by Scuola Normale Superiore, by INFN and by the MIUR-PRIN contract 2009KHZKRX.

\section{A Foregoing the traceless and transverse constraints}

In this appendix, we briefly discuss how the TT constraints can be forgone in a systematic fashion [44, 47]. We follow the discussion of the flat-space case, presented in Appendix B of [48], and sketch the generalization of the logic to (A)dS.

\footnotetext{
${ }^{19}$ See [78] for an analysis of flat-space gauge algebras.

${ }^{20}$ See $[21,22]$ for the free HS geometric equations, and [79] for a recent development on HS curvatures.

${ }^{21}$ See $[80,81]$ for the recent development of the free massive HS theory in the metric-like approach.

22 See e.g. [50, 82, 83] for some investigation along these lines.

${ }^{23}$ See [84-86] for the triplet system which contains the same DoFs as the massless limit of the first Regge trajectory of String Theory. See [87] for the analysis of HS interactions in a constant electromagnetic background within the String Theory framework. See [88-90] for the construction of some cubic and quartic flat-space vertices of massless HS fields using vertex operators in String theory, and [91] for its recent extension to AdS.

${ }^{24}$ In the $A d S_{3}$ case, there has been considerable recent development after the works [94, 95].
} 
Let us first recall the flat-space case. The idea is to start from the on-shell action and to compensate the outcome of the gauge variation that vanished with the TT constraints. For the quadratic part, one can see in this way that the action (2.3) can be completed as

$$
S^{(2)}=\frac{1}{2} \int d^{d} x e^{\partial_{u_{1}} \cdot \partial_{u_{2}}}\left(\partial_{x_{2}}^{2}+\mathcal{D}_{1} \mathcal{D}_{2}-\partial_{x_{2}}^{2} \mathcal{T}_{1} \mathcal{T}_{2}\right) \varphi\left(x_{1}, u_{1}\right) \varphi\left(x_{2}, u_{2}\right){\substack{x_{1}=x_{2}=x \\ u_{1}=u_{2}=0}}_{\substack{x_{2} \\ u_{1}}}
$$

where the operators $\mathcal{D}$ and $\mathcal{T}$ are defined as solutions of the following equations:

$$
\mathcal{D} \delta_{\varepsilon}^{(0)} \varphi(x, u)=\partial_{x}^{2} \varepsilon(x, u), \quad \mathcal{T} \delta_{\varepsilon}^{(0)} \varphi(x, u)=-\partial_{x} \cdot \partial_{u} \varepsilon(x, u),
$$

while $\mathcal{D}$ can be solved in terms of $\mathcal{T}$ as

$$
\mathcal{D}=\partial_{x} \cdot \partial_{u}+u \cdot \partial_{x} \mathcal{T} .
$$

The latter can be now obtained solving for the operator $\mathcal{T}$ that has to be found restricting the attention to operators that vanish with the TT constraints. One can recover the usual Fronsdal formulation [19] if we search for a solution for $\mathcal{T}$ built from traces without allowing for auxiliary fields. Indeed, at the end one recovers the following solution for $\mathcal{T}$ :

$$
\mathcal{T}=-\frac{1}{2} \partial_{u}^{2}
$$

that satisfies eq. (A.2) up to terms that are proportional to the trace of the gauge parameter. Since the gauge parameter is traceless, the double trace part of the field is gauge invariant. Hence, in order to be sure that after gauge fixing the original TT system is recovered, the double trace of the field must be set to zero. Let us mention that the different solutions for $\mathcal{T}$ provide other possible off-shell completions with the possibility of having different spectra. For instance, both the compensator system [23] and the triplet system [28, 29, 8486] can be recovered.

Let us now recall briefly how this procedure works for the flat-space cubic action. In order to simplify the discussion, we consider the simpler case in which the on-shell cubic coupling function is an exponential without any Chan-Paton factor:

$$
\mathcal{K}=e^{\ell \mathcal{V}}, \quad \mathcal{V}=\mathcal{Y}_{1}+\mathcal{Y}_{2}+\mathcal{Y}_{3}+\mathcal{G},
$$

where $\mathcal{Y}_{i}$ 's and $\mathcal{G}$ are defined in (2.11), and $\ell$ is a length parameter. The basic viewpoint is similar to that considered at the quadratic level and one needs to find the right counterterms to cure the non-zero gauge variation of the cubic action that is proportional to divergences. Moreover, gauge invariance should hold up to the Fronsdal equation:

$$
\left(\partial_{x}^{2}-u \cdot \partial_{x} \mathcal{D}\right) \varphi \approx 0
$$

since terms proportional to it can be compensated, and one obtains the following commutation relation and its cyclic permutations:

$$
\begin{aligned}
& {\left[\mathcal{V}, u_{1} \cdot \partial_{x_{1}}\right] \varphi_{1} \varphi_{2} \varepsilon_{3} \approx\left(\mathcal{H}_{23} u_{3} \cdot \partial_{x_{3}}-u_{2} \cdot \partial_{x_{2}} \mathcal{H}_{32}\right) \varphi_{1} \varphi_{2} \varepsilon_{3},} \\
& {\left[\mathcal{V}, u_{2} \cdot \partial_{x_{2}}\right] \varphi_{1} \varphi_{2} \varepsilon_{3} \approx\left(\mathcal{H}_{13} u_{1} \cdot \partial_{x_{1}}-u_{3} \cdot \partial_{x_{3}} \mathcal{H}_{31}\right) \varphi_{1} \varphi_{2} \varepsilon_{3},} \\
& {\left[\mathcal{V}, u_{3} \cdot \partial_{x_{3}}\right] \varphi_{1} \varphi_{2} \varepsilon_{3} \approx\left(u_{2} \cdot \partial_{x_{2}} \mathcal{H}_{12}-u_{1} \cdot \partial_{x_{1}} \mathcal{H}_{21}\right) \varphi_{1} \varphi_{2} \varepsilon_{3},}
\end{aligned}
$$


where

$$
\mathcal{H}_{i j}=\left(\partial_{u_{i}} \cdot \partial_{u_{j}}+1\right) \mathcal{D}_{j}+\partial_{u_{i}} \cdot \partial_{x_{j}} \mathcal{T}_{j}
$$

This set of commutators encodes a recursive structure from which one can reconstruct the full off-shell completion of the cubic vertex. The end result can be expressed in the following compact form:

$$
\mathcal{K}^{\text {off-shell }}=e^{\ell \mathcal{V}}\left[1+\ell^{2} \mathcal{H}_{12} \mathcal{H}_{13}+\ell^{3}: \mathcal{H}_{21} \mathcal{H}_{32} \mathcal{H}_{13}:+(\text { cyclic perm. })\right],
$$

where : : enforces an ordering in which the generalized de Donder operators are to act directly on the fields and hence are to be put to the right:

$$
: \mathcal{D}_{1} \mathcal{Z}_{2}:=\mathcal{Z}_{2} \mathcal{D}_{1}
$$

The (A)dS off-shell completion is expected to work on very similar grounds. In the following, for simplicity, we consider the quadratic part and then sketch how this procedure extends to the cubic level. The only complication of $(\mathrm{A}) \mathrm{dS}$ is the presence of the $\delta$-function in the action, that requires in principle further counterterms in order to compensate for the various total derivatives that are generated. The starting point is now eq. (3.22) and the only additional terms in the gauge variation that are to be considered are those proportional to a total derivative and are of the form:

$$
\delta S^{(2)}=\left.\int \frac{d^{d+1} X}{L} \delta\left(\frac{\sqrt{X^{2}}}{L}-1\right) \partial_{X} \cdot \partial_{U_{2}} e^{\partial_{U_{1}} \cdot \partial_{U_{2}}} \partial_{X_{2}}^{2} E\left(X_{1}, U_{1}\right) \Phi\left(X_{2}, U_{2}\right)\right|_{\substack{X_{1}=X_{2}=X \\ U_{1}=U_{2}=0}} .
$$

This additional term, after an integration by parts, gives $X_{1} \cdot \partial_{U_{1}}$ and vanishes with the tangent condition (3.11). Hence, the off-shell ambient-space action for totally symmetric (A)dS fields is

$$
\begin{aligned}
S^{(2)}=\frac{1}{2} \int & \frac{d^{d+1} X}{L} \delta\left(\frac{\sqrt{X^{2}}}{L}-1\right) \times \\
& \times\left. e^{\partial_{U_{1}} \cdot \partial_{U_{2}}}\left(\partial_{X_{2}}^{2}+\mathcal{D}_{1} \mathcal{D}_{2}-\partial_{X_{2}}^{2} \mathcal{T}_{1} \mathcal{T}_{2}\right) \Phi\left(X_{1}, U_{1}\right) \Phi\left(X_{2}, U_{2}\right)\right|_{\substack{X_{1}=X_{2}=X \\
U_{1}=U_{2}=0}}
\end{aligned}
$$

The same procedure that we have considered in the simple quadratic case has to be performed also at the cubic order. Starting from the ambient-space analogue of the off-shell cubic vertex $S^{(3)}$ (A.9), one gets the following schematic form of the gauge variation

$$
\begin{aligned}
\delta S^{(3)}=\int d^{d+1} X & \sum_{n=1}^{\infty} \delta^{(n)}\left(\frac{\sqrt{X^{2}}}{L}-1\right) \times \\
& \times\left.\partial_{X_{i}} \cdot \partial_{U_{i}}(\cdots) E\left(X_{1}, U_{1}\right) \Phi\left(X_{2}, U_{2}\right) \Phi\left(X_{3}, U_{3}\right)\right|_{\substack{X_{1}=X_{2}=x_{3}=X \\
U_{1}=U_{2}=U_{3}=0}},
\end{aligned}
$$

that is to be compensated adding further divergence and trace terms at the $\delta^{(1)}$-level. This procedure is expected to work order by order, so leading eventually to the off-shell form of the (A)dS cubic action. 


\section{B Proof at the $\delta^{(2)}$ level}

In this section, we prove that the total-derivative part $\mathcal{C}$ in (4.28) does not impose additional conditions on the constants $\alpha_{i}$ and $\beta_{i}$. At the level of $\delta^{(1)}, \mathcal{C}$ does not vanish with (4.17 4.18), but is simplified to

$$
\begin{aligned}
\mathcal{C}= & \left(\alpha_{1}^{2}-1\right) \partial_{X} \cdot \partial_{U_{1}}+2\left(\alpha_{2}+1\right) \partial_{X} \cdot \partial_{U_{2}}-2\left(\alpha_{3}-1\right) \partial_{X} \cdot \partial_{U_{3}} \\
& -\left(\alpha_{1}-1\right)\left(\beta_{3}-\frac{1}{2}\right) \partial_{X} \cdot \partial_{U_{2}} \partial_{U_{3}} \cdot \partial_{U_{1}}-\left(\alpha_{1}+1\right)\left(\beta_{2}+\frac{1}{2}\right) \partial_{X} \cdot \partial_{U_{3}} \partial_{U_{1}} \cdot \partial_{U_{2}} \\
& +\left[2\left(\alpha_{1} \beta_{1}-1\right) \partial_{X} \cdot \partial_{U_{1}}+\frac{3}{2}\left(\alpha_{2}+1\right) \partial_{X} \cdot \partial_{U_{2}}-\frac{3}{2}\left(\alpha_{3}-1\right) \partial_{X} \cdot \partial_{U_{3}}\right] \partial_{U_{2}} \cdot \partial_{U_{3}} \\
& +\left[\left(\beta_{1}^{2}-1\right) \partial_{X} \cdot \partial_{U_{1}} \partial_{U_{2}} \cdot \partial_{U_{3}}-\left(\beta_{3}-\frac{1}{2}\right)\left(\beta_{1}+\beta_{2}\right) \partial_{X} \cdot \partial_{U_{2}} \partial_{U_{3}} \cdot \partial_{U_{1}}\right. \\
& \left.\quad-\left(\beta_{2}+\frac{1}{2}\right)\left(\beta_{3}+\beta_{1}\right) \partial_{X} \cdot \partial_{U_{3}} \partial_{U_{1}} \cdot \partial_{U_{2}}\right] \partial_{U_{2}} \cdot \partial_{U_{3}} \cdot
\end{aligned}
$$

We integrate by parts in order to replace $\delta^{(1)} \partial_{X} \cdot \partial_{U_{i}}$ with $-\delta^{(2)} X_{i} \cdot \partial_{U_{i}} / L^{2}$, and then $\delta^{(1)} \mathcal{C}$ can be rewritten as $-\delta^{(2)} \mathcal{D} / L^{2}$ with $\mathcal{D}$ some other differential operator. We now push $\mathcal{D}$ to the right hand side of $e^{L \mathcal{V}}$ as

$$
\int \delta^{(2)} k \mathcal{D} e^{L \mathcal{V}} E_{1} \Phi_{2} \Phi_{3}\left|=\int \delta^{(2)} k e^{L \mathcal{V}} L \mathcal{E} E_{1} \Phi_{2} \Phi_{3}\right|,
$$

getting the following operator acting on the fields:

$$
\begin{aligned}
\mathcal{E}=\{ & -\left[2\left(\alpha_{1} \beta_{1}-1\right)\left(\alpha_{2}-1\right)-\left(\alpha_{1}+1\right)\left(\alpha_{2}+1\right)\left(\beta_{2}-1\right)+2\left(\alpha_{2}+1\right)\left(\beta_{3}+\beta_{1}\right)\right] \partial_{U_{1}} \cdot \partial_{U_{2}} \\
& -\left[2\left(\alpha_{1} \beta_{1}-1\right)\left(\alpha_{3}+1\right)-\left(\alpha_{3}-1\right)\left(\alpha_{1}-1\right)\left(\beta_{3}+1\right)+2\left(\alpha_{3}-1\right)\left(\beta_{1}+\beta_{2}\right)\right] \partial_{U_{3}} \cdot \partial_{U_{1}} \\
& -\left[-\left(\alpha_{2}-1\right)\left(\beta_{1}^{2}-1\right)+\left(\alpha_{2}+1\right)\left(\beta_{2}-1\right)\left(\beta_{3}+\beta_{1}\right)\right] \partial_{U_{1}} \cdot \partial_{U_{2}} \partial_{U_{2}} \cdot \partial_{U_{3}} \\
& -\left[-\left(\alpha_{3}+1\right)\left(\beta_{1}^{2}-1\right)+\left(\alpha_{3}-1\right)\left(\beta_{3}+1\right)\left(\beta_{1}+\beta_{2}\right)\right] \partial_{U_{3}} \cdot \partial_{U_{1}} \partial_{U_{2}} \cdot \partial_{U_{3}} \\
& \left.+\left(\beta_{2}+\beta_{3}\right)\left[\alpha_{1}\left(\beta_{2}+\beta_{3}\right)+\beta_{2}-\beta_{3}+2\right] \partial_{U_{1}} \cdot \partial_{U_{2}} \partial_{U_{3}} \cdot \partial_{U_{1}}\right\} \partial_{U_{2}} \cdot \partial_{U_{3}} \cdot
\end{aligned}
$$

None of these contributions can be compensated, so that each coefficient in the above formula should vanish separately. Using the general solutions (4.20) of (4.17 - 4.19), one can verify that this is indeed the case.

\section{Radial reduction of the 3-3-2 vertex}

In this Appendix we present more details of the reduction of the 3-3-2 vertex (4.41) to the (A)dS-intrinsic expression (4.49). Expanding the operator in eq. (4.41) gives altogether six terms:

$$
\begin{aligned}
& {\left[\partial_{U_{2}} \cdot \partial_{U_{3}} \partial_{U_{1}} \cdot \partial_{X_{2}}-\partial_{U_{1}} \cdot \partial_{U_{3}} \partial_{U_{2}} \cdot \partial_{X_{1}}+\frac{1}{2} \partial_{U_{1}} \cdot \partial_{U_{2}} \partial_{U_{3}} \cdot \partial_{X_{12}}\right]^{2} \partial_{U_{1}} \cdot \partial_{X_{2}} \partial_{U_{2}} \cdot \partial_{X_{1}}} \\
& =\partial_{U_{2}} \cdot \partial_{U_{3}}\left(\partial_{U_{1}} \cdot \partial_{X_{2}}\right)^{3} \partial_{U_{2}} \cdot \partial_{X_{1}}+(1 \leftrightarrow 2) \\
& \quad-2 \partial_{U_{1}} \cdot \partial_{U_{3}} \partial_{U_{2}} \cdot \partial_{U_{3}}\left(\partial_{U_{1}} \cdot \partial_{X_{2}}\right)^{2}\left(\partial_{U_{2}} \cdot \partial_{X_{1}}\right)^{2} \\
& \quad+\partial_{U_{1}} \cdot \partial_{U_{2}} \partial_{U_{2}} \cdot \partial_{U_{3}}\left(\partial_{U_{1}} \cdot \partial_{X_{2}}\right)^{2} \partial_{X_{1}} \cdot \partial_{U_{2}} \partial_{U_{3}} \cdot \partial_{X_{12}}+(1 \leftrightarrow 2) \\
& \quad+\frac{1}{4}\left(\partial_{U_{1}} \cdot \partial_{U_{2}}\right)^{2} \partial_{U_{1}} \cdot \partial_{X_{2}} \partial_{U_{2}} \cdot \partial_{X_{1}}\left(\partial_{U_{3}} \cdot \partial_{X_{12}}\right)^{2}
\end{aligned}
$$

but taking into account the symmetries under $1 \leftrightarrow 2$, one is left with four terms. One of such terms is (4.43), and we have sketched how to get the corresponding (A)dS intrinsic 
expression (4.48). Applying the same techniques explained there, one can deal with the other three terms in the same manner.

We present the (A)dS intrinsic expression for each term. The third term in the expansion (C.1) gives

$$
\begin{aligned}
& \partial_{U_{1}} \cdot \partial_{U_{3}} \partial_{U_{2}} \cdot \partial_{U_{3}}\left(\partial_{X_{2}} \cdot \partial_{U_{1}}\right)^{2}\left(\partial_{X_{1}} \cdot \partial_{U_{2}}\right)^{2} \\
& \simeq \partial_{u_{1}} \cdot \partial_{u_{3}} \partial_{u_{2}} \cdot \partial_{u_{3}}\left(\partial_{u_{1}} \cdot D_{2}\right)^{2}\left(\partial_{u_{2}} \cdot D_{1}\right)^{2}+\frac{1}{L^{2}} \partial_{u_{1}} \cdot \partial_{u_{2}} \partial_{u_{1}} \cdot \partial_{u_{3}} \partial_{u_{2}} \cdot \partial_{u_{3}} \partial_{u_{1}} \cdot D_{2} \partial_{u_{2}} \cdot D_{1} \\
& \quad-\frac{1}{L^{2}} \partial_{u_{1}} \cdot \partial_{u_{2}}\left(\partial_{u_{1}} \cdot \partial_{u_{3}}\right)^{2}\left(\partial_{u_{2}} \cdot D_{1}\right)^{2}-\frac{1}{L^{2}} \partial_{u_{1}} \cdot \partial_{u_{2}}\left(\partial_{u_{2}} \cdot \partial_{u_{3}}\right)^{2}\left(\partial_{u_{1}} \cdot D_{2}\right)^{2} \\
& \quad+\frac{d+4}{L^{4}}\left(\partial_{u_{1}} \cdot \partial_{u_{2}}\right)^{2} \partial_{u_{1}} \cdot \partial_{u_{3}} \partial_{u_{2}} \cdot \partial_{u_{3}},
\end{aligned}
$$

where $\simeq$ means equivalence of two operators under the condition (4.46). The fourth term gives

$$
\begin{aligned}
& \partial_{U_{1}} \cdot \partial_{U_{2}} \partial_{U_{2}} \cdot \partial_{U_{3}}\left(\partial_{U_{1}} \cdot \partial_{X_{2}}\right)^{2} \partial_{U_{2}} \cdot \partial_{X_{1}} \partial_{U_{3}} \cdot \partial_{X_{12}} \\
& \simeq \partial_{u_{1}} \cdot \partial_{u_{2}} \partial_{u_{2}} \cdot \partial_{u_{3}}\left(\partial_{u_{1}} \cdot D_{2}\right)^{2} \partial_{u_{2}} \cdot D_{1} \partial_{u_{3}} \cdot D_{12}+\frac{1}{L^{2}} \partial_{u_{1}} \cdot \partial_{u_{2}}\left(\partial_{u_{2}} \cdot \partial_{u_{3}}\right)^{2}\left(\partial_{u_{1}} \cdot D_{2}\right)^{2} \\
& \quad-\frac{1}{L^{2}}\left(\partial_{u_{1}} \cdot \partial_{u_{2}}\right)^{2} \partial_{u_{1}} \cdot \partial_{u_{3}} \partial_{u_{2}} \cdot D_{1} \partial_{u_{3}} \cdot D_{12}+\frac{2}{L^{2}}\left(\partial_{u_{1}} \cdot \partial_{u_{2}}\right)^{2} \partial_{u_{2}} \cdot \partial_{u_{3}} \partial_{u_{1}} \cdot D_{2} \partial_{u_{3}} \cdot D_{12} \\
& \quad+\frac{3}{L^{2}} \partial_{u_{1}} \cdot \partial_{u_{2}} \partial_{u_{1}} \cdot \partial_{u_{3}} \partial_{u_{2}} \cdot \partial_{u_{3}} \partial_{u_{1}} \cdot D_{2} \partial_{u_{2}} \cdot D_{1}-\frac{d+1}{L^{4}}\left(\partial_{u_{1}} \cdot \partial_{u_{2}}\right)^{2} \partial_{u_{1}} \cdot \partial_{u_{3}} \partial_{u_{2}} \cdot \partial_{u_{3}},
\end{aligned}
$$

and the fifth term can be obtained interchanging 1 and 2 in the above. The last term gives

$$
\begin{aligned}
& \left(\partial_{U_{1}} \cdot \partial_{U_{2}}\right)^{2} \partial_{U_{1}} \cdot \partial_{X_{2}} \partial_{U_{2}} \cdot \partial_{X_{1}}\left(\partial_{U_{3}} \cdot \partial_{X_{12}}\right)^{2} \\
& \simeq \partial_{u_{1}} \cdot D_{2}\left(\partial_{u_{2}} \cdot D_{12}\right)^{2}+\frac{2}{L^{2}}\left(\partial_{u_{1}} \cdot \partial_{u_{2}}\right)^{3}\left(\partial_{u_{3}} \cdot D_{12}\right)^{2} \\
& \quad-\frac{5}{L^{2}} \partial_{u_{1}} \cdot \partial_{u_{3}}\left(\partial_{u_{1}} \cdot \partial_{u_{2}}\right)^{2} \partial_{u_{2}} \cdot D_{1} \partial_{u_{3}} \cdot D_{2}-\frac{5}{L^{2}} \partial_{u_{2}} \cdot \partial_{u_{3}}\left(\partial_{u_{1}} \cdot \partial_{u_{2}}\right)^{2} \partial_{u_{1}} \cdot D_{2} \partial_{u_{3}} \cdot D_{1} \\
& \quad-\frac{8}{L^{2}} \partial_{u_{1}} \cdot \partial_{u_{2}} \partial_{u_{1}} \cdot \partial_{u_{3}} \partial_{u_{2}} \cdot \partial_{u_{3}} \partial_{u_{1}} \cdot D_{2} \partial_{u_{2}} \cdot D_{1}+2 \frac{d-9}{L^{4}}\left(\partial_{u_{1}} \cdot \partial_{u_{2}}\right)^{2} \partial_{u_{1}} \cdot \partial_{u_{3}} \partial_{u_{2}} \cdot \partial_{u_{3}},
\end{aligned}
$$

and collecting all these terms finally gives (4.49).

\section{References}

[1] R. Argurio, G. Barnich, G. Bonelli, and M. Grigoriev, eds., "Higher-Spin Gauge Theories", Proceedings of the First Solvay Workshop, held in Brussels on May 12-14, 2004, Int. Solvay Institutes, 2006.

[2] M. Bianchi and V. Didenko, Massive higher spin multiplets and holography, hep-th/0502220.

[3] D. Francia and C. Hull, Higher-spin gauge fields and duality, hep-th/0501236.

[4] N. Bouatta, G. Compere, and A. Sagnotti, An Introduction to free higher-spin fields, hep-th/0409068.

[5] X. Bekaert, S. Cnockaert, C. Iazeolla, and M. Vasiliev, Nonlinear higher spin theories in various dimensions, hep-th/0503128.

[6] A. Sagnotti, E. Sezgin, and P. Sundell, On higher spins with a strong $S p(2, R)$ condition, hep-th/0501156.

[7] D. Sorokin, Introduction to the classical theory of higher spins, AIP Conf.Proc. 767 (2005) 172-202, [hep-th/0405069]. 
[8] D. Francia and A. Sagnotti, Higher-spin geometry and string theory, J. Phys. Conf. Ser. 33 (2006) 57, [hep-th/0601199].

[9] X. Bekaert, N. Boulanger, and P. Sundell, How higher-spin gravity surpasses the spin two barrier: no-go theorems versus yes-go examples, arXiv:1007.0435.

[10] M. A. Vasiliev, Consistent equations for interacting massless fields of all spins in the first order in curvatures, Annals Phys. 190 (1989) 59-106.

[11] M. Vasiliev, Nonlinear equations for symmetric massless higher spin fields in (A)dS(d), Phys.Lett. B567 (2003) 139-151, [hep-th/0304049].

[12] M. Vasiliev, Higher spin gauge theories in various dimensions, Fortsch. Phys. 52 (2004) 702-717, [hep-th/0401177].

[13] N. Boulanger and P. Sundell, An action principle for Vasiliev's four-dimensional higher-spin gravity, arXiv:1102.2219.

[14] N. Colombo and P. Sundell, Twistor space observables and quasi-amplitudes in $4 D$ higher spin gravity, arXiv: 1012.0813.

[15] E. Sezgin and P. Sundell, Geometry and Observables in Vasiliev's Higher Spin Gravity, arXiv: 1103.2360.

[16] V. Didenko, A. Matveev, and M. Vasiliev, Unfolded Description of AdS(4) Kerr Black Hole, Phys.Lett. B665 (2008) 284-293, [arXiv:0801.2213].

[17] C. Iazeolla, On the Algebraic Structure of Higher-Spin Field Equations and New Exact Solutions, arXiv:0807.0406.

[18] C. Iazeolla and P. Sundell, Families of exact solutions to Vasiliev's 4 D equations with spherical, cylindrical and biaxial symmetry, arXiv:1107.1217.

[19] C. Fronsdal, Massless Fields with Integer Spin, Phys. Rev. D18 (1978) 3624.

[20] J. Fang and C. Fronsdal, Massless Fields with Half Integral Spin, Phys. Rev. D18 (1978) 3630 .

[21] D. Francia and A. Sagnotti, Free geometric equations for higher spins, Phys. Lett. B543 (2002) 303-310, [hep-th/0207002].

[22] D. Francia and A. Sagnotti, On the geometry of higher spin gauge fields, Class. Quant. Grav. 20 (2003) S473-S486, [hep-th/0212185].

[23] D. Francia and A. Sagnotti, Minimal local Lagrangians for higher-spin geometry, Phys. Lett. B624 (2005) 93-104, [hep-th/0507144].

[24] A. Campoleoni, D. Francia, J. Mourad, and A. Sagnotti, Unconstrained Higher Spins of Mixed Symmetry. I. Bose Fields, Nucl. Phys. B815 (2009) 289-367, [arXiv:0810.4350].

[25] A. Campoleoni, D. Francia, J. Mourad, and A. Sagnotti, Unconstrained Higher Spins of Mixed Symmetry. II. Fermi Fields, Nucl. Phys. B828 (2010) 425, [arXiv:0904.4447].

[26] A. Campoleoni, Lagrangian formulations for Bose and Fermi higher-spin fields of mixed symmetry, arXiv:0905.1472.

[27] A. Campoleoni, Metric-like Lagrangian Formulations for Higher-Spin Fields of Mixed Symmetry, Riv.Nuovo Cim. 033 (2010) 123-253, [arXiv:0910.3155].

[28] D. Francia, On the relation between local and geometric Lagrangians for higher spins, J.Phys.Conf.Ser. 222 (2010) 012002, [arXiv:1001.3854]. 
[29] D. Francia, String theory triplets and higher-spin curvatures, Phys.Lett. B690 (2010) 90-95, [arXiv: 1001.5003].

[30] D. Francia, Low-spin models for higher-spin Lagrangians, Prog.Theor.Phys.Suppl. 188 (2011) 94-105, [arXiv: 1103.0683].

[31] E. Fradkin and M. A. Vasiliev, Cubic Interaction in Extended Theories of Massless Higher Spin Fields, Nucl. Phys. B291 (1987) 141.

[32] E. Fradkin and M. A. Vasiliev, On the Gravitational Interaction of Massless Higher Spin Fields, Phys. Lett. B189 (1987) 89-95.

[33] M. Vasiliev, Cubic Vertices for Symmetric Higher-Spin Gauge Fields in $(A) d S_{d}$, arXiv: 1108.5921.

[34] K. Alkalaev, FV-type action for AdS $S_{5}$ mixed-symmetry fields, JHEP 1103 (2011) 031, [arXiv: 1011.6109].

[35] Y. Zinoviev, Spin 3 cubic vertices in a frame-like formalism, JHEP 1008 (2010) 084, [arXiv: 1007.0158].

[36] N. Boulanger, E. Skvortsov, and Y. Zinoviev, Gravitational cubic interactions for a simple mixed-symmetry gauge field in AdS and flat backgrounds, J.Phys.A A44 (2011) 415403, [arXiv:1107.1872].

[37] N. Boulanger and E. Skvortsov, Higher-spin algebras and cubic interactions for simple mixed-symmetry fields in AdS spacetime, JHEP 1109 (2011) 063, [arXiv:1107.5028].

[38] F. A. Berends, G. Burgers, and H. van Dam, On the theoretical problems in constructing interactions involving higher spin massless particles, Nucl. Phys. B260 (1985) 295.

[39] F. A. Berends, G. Burgers, and H. van Dam, Explicit construction of conserved currents for massless fields of arbitrary spin, Nucl. Phys. B271 (1986) 429.

[40] E. Fradkin and R. Metsaev, Cubic scattering amplitudes for all massless representations of the Poincare group in any space-time dimension, Phys.Rev. D52 (1995) 4660-4667.

[41] R. Metsaev, Cubic interaction vertices of massive and massless higher spin fields, Nucl.Phys. B759 (2006) 147-201, [hep-th/0512342].

[42] R. Metsaev, Cubic interaction vertices for fermionic and bosonic arbitrary spin fields, arXiv:0712.3526.

[43] R. Manvelyan, K. Mkrtchyan, and W. Ruehl, Direct construction of a cubic selfinteraction for higher spin gauge fields, Nucl.Phys. B844 (2011) 348-364, [arXiv:1002.1358].

[44] R. Manvelyan, K. Mkrtchyan, and W. Ruehl, General trilinear interaction for arbitrary even higher spin gauge fields, Nucl. Phys. B836 (2010) 204-221, [arXiv:1003.2877].

[45] K. Mkrtchyan, Higher Spin Interacting Quantum Field Theory and Higher Order Conformal Invariant Lagrangians, arXiv:1011.0160. Ph.D. Thesis. Advisor Prof. Ruben Manvelyan.

[46] M. Taronna, Higher Spins and String Interactions, arXiv:1005.3061.

[47] A. Sagnotti and M. Taronna, String Lessons for Higher-Spin Interactions, Nucl. Phys. B842 (2011) 299-361, [arXiv:1006.5242].

[48] M. Taronna, Higher-Spin Interactions: four-point functions and beyond, arXiv:1107.5843.

[49] A. Fotopoulos and M. Tsulaia, On the Tensionless Limit of String theory, Off - Shell Higher Spin Interaction Vertices and BCFW Recursion Relations, arXiv:1009.0727. 
[50] Y. M. Zinoviev, On spin 3 interacting with gravity, Class. Quant. Grav. 26 (2009) 035022, [arXiv:0805.2226].

[51] N. Boulanger, S. Leclercq, and P. Sundell, On The Uniqueness of Minimal Coupling in Higher-Spin Gauge Theory, JHEP 0808 (2008) 056, [arXiv: 0805.2764].

[52] R. Manvelyan and K. Mkrtchyan, Conformal invariant interaction of a scalar field with the higher spin field in AdS(D), Mod.Phys.Lett. A25 (2010) 1333-1348, [arXiv:0903.0058].

[53] A. Fotopoulos, N. Irges, A. C. Petkou, and M. Tsulaia, Higher-Spin Gauge Fields Interacting with Scalars: The Lagrangian Cubic Vertex, JHEP 0710 (2007) 021, [arXiv:0708.1399].

[54] I. Buchbinder, A. Fotopoulos, A. C. Petkou, and M. Tsulaia, Constructing the cubic interaction vertex of higher spin gauge fields, Phys.Rev. D74 (2006) 105018, [hep-th/0609082].

[55] A. Fotopoulos and M. Tsulaia, Gauge Invariant Lagrangians for Free and Interacting Higher Spin Fields. A Review of the BRST formulation, Int.J.Mod.Phys. A24 (2009) 1-60, [arXiv:0805.1346]. Extended version of the contribution to the volume dedicated of Prof I.L. Buchbinder.

[56] A. Fotopoulos and M. Tsulaia, Current Exchanges for Reducible Higher Spin Modes on AdS, arXiv: 1007.0747.

[57] X. Bekaert and E. Meunier, Higher spin interactions with scalar matter on constant curvature spacetimes: conserved current and cubic coupling generating functions, JHEP 11 (2010) 116, [arXiv:1007.4384].

[58] D. Francia, J. Mourad, and A. Sagnotti, Current Exchanges and Unconstrained Higher Spins, Nucl. Phys. B773 (2007) 203-237, [hep-th/0701163].

[59] A. Sagnotti, Higher Spins and Current Exchanges, arXiv:1002.3388.

[60] X. Bekaert, E. Joung, and J. Mourad, On higher spin interactions with matter, JHEP 0905 (2009) 126, [arXiv:0903.3338].

[61] X. Bekaert, E. Joung, and J. Mourad, Effective action in a higher-spin background, JHEP 1102 (2011) 048, [arXiv: 1012.2103].

[62] C. Fronsdal, Singletons and Massless, Integral Spin Fields on de Sitter Space (Elementary Particles in a Curved Space. 7.), Phys. Rev. D20 (1979) 848-856.

[63] R. Manvelyan, K. Mkrtchyan, and W. Ruehl, A generating function for the cubic interactions of higher spin fields, Phys. Lett. B696 (2011) 410-415, [arXiv:1009.1054].

[64] S. Deser and R. Arnowitt, Quantum Theory of Gravitation I-Linearized Theory, Phys.Rev. 113 (1959) 745.

[65] R. Metsaev, Massless mixed symmetry bosonic free fields in d-dimensional anti-de Sitter space-time, Phys.Lett. B354 (1995) 78-84.

[66] R. Metsaev, Arbitrary spin massless bosonic fields in d-dimensional anti-de Sitter space, hep-th/9810231.

[67] T. Biswas and W. Siegel, Radial dimensional reduction: Anti-de Sitter theories from flat, JHEP 0207 (2002) 005, [hep-th/0203115].

[68] X. Bekaert, I. Buchbinder, A. Pashnev, and M. Tsulaia, On higher spin theory: Strings, BRST, dimensional reductions, Class. Quant. Grav. 21 (2004) S1457-1464, 
[hep-th/0312252].

[69] K. Hallowell and A. Waldron, Constant curvature algebras and higher spin action generating functions, Nucl.Phys. B724 (2005) 453-486, [hep-th/0505255].

[70] G. Barnich and M. Grigoriev, Parent form for higher spin fields on anti-de Sitter space, JHEP 0608 (2006) 013, [hep-th/0602166].

[71] A. Fotopoulos, K. L. Panigrahi, and M. Tsulaia, Lagrangian formulation of higher spin theories on AdS space, Phys. Rev. D74 (2006) 085029, [hep-th/0607248].

[72] D. Francia, J. Mourad, and A. Sagnotti, (A)dS exchanges and partially-massless higher spins, Nucl. Phys. B804 (2008) 383-420, [arXiv:0803.3832].

[73] N. Boulanger, C. Iazeolla, and P. Sundell, Unfolding Mixed-Symmetry Fields in AdS and the BMV Conjecture: I. General Formalism, JHEP 0907 (2009) 013, [arXiv:0812.3615].

[74] N. Boulanger, C. Iazeolla, and P. Sundell, Unfolding Mixed-Symmetry Fields in AdS and the BMV Conjecture. II. Oscillator Realization, JHEP 0907 (2009) 014, [arXiv:0812.4438].

[75] K. B. Alkalaev and M. Grigoriev, Unified BRST description of AdS gauge fields, Nucl.Phys. B835 (2010) 197-220, [arXiv:0910.2690].

[76] N. Boulanger, S. Leclercq, and S. Cnockaert, Parity violating vertices for spin-3 gauge fields, Phys. Rev. D73 (2006) 065019, [hep-th/0509118].

[77] A. Sagnotti, The higher-spin challenge, in Strings 2009, 2009. [http://strings2009.roma2.infn.it].

[78] X. Bekaert, N. Boulanger, and S. Leclercq, Strong obstruction of the Berends-Burgers-van Dam spin-3 vertex, J. Phys. A A43 (2010) 185401, [arXiv:1002.0289].

[79] R. Manvelyan, K. Mkrtchyan, W. Ruhl, and M. Tovmasyan, On Nonlinear Higher Spin Curvature, Phys.Lett. B699 (2011) 187-191, [arXiv:1102.0306].

[80] D. Francia, Geometric Lagrangians for massive higher-spin fields, Nucl. Phys. B796 (2008) 77-122, [arXiv:0710.5378].

[81] D. Francia, Geometric massive higher spins and current exchanges, Fortsch. Phys. 56 (2008) 800-808, [arXiv:0804.2857].

[82] Y. Zinoviev, On spin 2 electromagnetic interactions, Mod.Phys.Lett. A24 (2009) 17-23, [arXiv:0806.4030].

[83] Y. Zinoviev, On massive spin 2 electromagnetic interactions, Nucl.Phys. B821 (2009) 431-451, [arXiv: 0901.3462].

[84] A. K. Bengtsson, A Unified Action For Higher Spin Gauge Bosons From Covariant String Theory, Phys.Lett. B182 (1986) 321.

[85] M. Henneaux and C. Teitelboim, Quantum Mechanics of Fundamental System, 2. Plenum Press, Newyork, 1987.

[86] A. Sagnotti and M. Tsulaia, On higher spins and the tensionless limit of string theory, Nucl.Phys. B682 (2004) 83-116, [hep-th/0311257].

[87] M. Porrati, R. Rahman, and A. Sagnotti, String Theory and The Velo-Zwanziger Problem, Nucl.Phys. B846 (2011) 250-282, [arXiv:1011.6411].

[88] D. Polyakov, Interactions of Massless Higher Spin Fields From String Theory, Phys. Rev. D82 (2010) 066005, [arXiv:0910.5338]. 
[89] D. Polyakov, Gravitational Couplings of Higher Spins from String Theory, Int. J. Mod. Phys. A25 (2010) 4623-4640, [arXiv: 1005.5512].

[90] D. Polyakov, Higher Spins and Open Strings: Quartic Interactions, Phys.Rev. D83 (2011) 046005, [arXiv: 1011.0353].

[91] D. Polyakov, A String Model for AdS Gravity and Higher Spins, arXiv:1106.1558.

[92] E. Sezgin and P. Sundell, Massless higher spins and holography, Nucl. Phys. B644 (2002) 303-370, [hep-th/0205131].

[93] I. Klebanov and A. Polyakov, AdS dual of the critical $O(N)$ vector model, Phys. Lett. B550 (2002) 213-219, [hep-th/0210114].

[94] A. Campoleoni, S. Fredenhagen, S. Pfenninger, and S. Theisen, Asymptotic symmetries of three-dimensional gravity coupled to higher-spin fields, JHEP 1011 (2010) 007, [arXiv: 1008.4744].

[95] M. Henneaux and S.-J. Rey, Nonlinear $W_{\text {infinity }}$ as Asymptotic Symmetry of Three-Dimensional Higher Spin Anti-de Sitter Gravity, JHEP 1012 (2010) 007, [arXiv: 1008.4579]. 